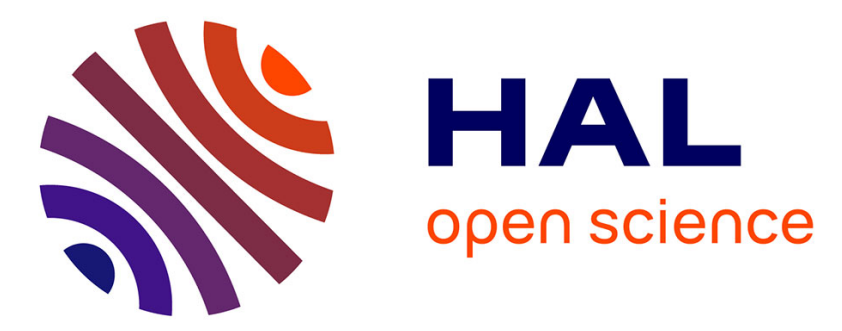

\title{
Understanding and Design of Bidirectional and Reversible Catalysts of Multielectron, Multistep Reactions
}

Vincent Fourmond, Eric S Wiedner, Wendy J Shaw, Christophe Léger

\section{To cite this version:}

Vincent Fourmond, Eric S Wiedner, Wendy J Shaw, Christophe Léger. Understanding and Design of Bidirectional and Reversible Catalysts of Multielectron, Multistep Reactions. Journal of the American Chemical Society, 2019, 10.1021/jacs.9b04854 . hal-02179792

\section{HAL Id: hal-02179792 \\ https://hal-amu.archives-ouvertes.fr/hal-02179792}

Submitted on 11 Jul 2019

HAL is a multi-disciplinary open access archive for the deposit and dissemination of scientific research documents, whether they are published or not. The documents may come from teaching and research institutions in France or abroad, or from public or private research centers.
L'archive ouverte pluridisciplinaire HAL, est destinée au dépôt et à la diffusion de documents scientifiques de niveau recherche, publiés ou non, émanant des établissements d'enseignement et de recherche français ou étrangers, des laboratoires publics ou privés.

\section{(1) (1) $\$$}

Distributed under a Creative Commons Attribution - NonCommercial - NoDerivatives| 4.0 


\title{
On the understanding and design of bidirectional and reversible catalysts of multielectron, multistep reactions
}

\author{
Vincent Fourmond, ${ }^{*}$, a Eric S. Wiedner, ${ }^{b}$ Wendy J. Shaw, ${ }^{\mathrm{b}}$ Christophe Léger ${ }^{*}$ a \\ a Aix Marseille Univ., CNRS, BIP UMR 7281, Marseille, France

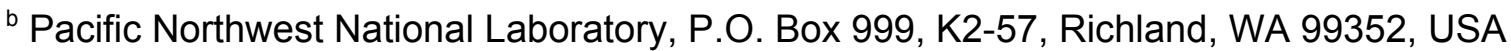

\section{Abstract}

Some enzymes, including those that are involved in the activation of small molecules such as $\mathrm{H}_{2}$ or $\mathrm{CO}_{2}$, can be wired to electrodes and function in either direction of the reaction depending on the electrochemical driving force, and display a significant rate at very small deviations from the equilibrium potential. We call the former property "bidirectionality", and the latter "reversibility". This performance sets very high standards for chemists who aim at designing synthetic electrocatalysts. Only recently, in the particular case of the hydrogen production/evolution reaction, has it been possible to produce inorganic catalysts that function bidirectionally, with an even smaller number that also function reversibly. This raises the question of how to engineer such desirable properties in other synthetic catalysts. Here we introduce the kinetic modelling of bidirectional two-electron redox reactions in the case of molecular catalysts and enzymes that are either attached to an electrode or diffusing in solution in the vicinity of an electrode. We emphasize that trying to discuss bidirectionality and reversibility in relation to a single redox potential leads to an impasse: the catalyst undergoes two redox transitions, therefore two catalytic potentials must be defined, which may depart from the two potentials measured in the absence of catalysis. The difference between the two catalytic potentials defines the reversibility; the difference between their average value and the equilibrium potential defines the directionality (also called "preference", or "bias"). We describe how the sequence of events in the bidirectional catalytic cycle can be elucidated based on the voltammetric responses. Further we discuss the design principles of bidirectionality and reversibility in terms of thermodynamics and kinetics, and conclude that neither bidirectionality nor reversibility requires that the catalytic energy landscape be flat. These theoretical findings are illustrated by previous results obtained with nickel diphosphine molecular catalysts and hydrogenases. In particular, analysis of the nickel catalysts highlights the fact that reversible catalysis can be achieved by catalysts that follow complex mechanisms with branched reaction pathways. 


\section{Introduction}

In addition to structural and spectroscopic methods, electrochemistry has proved invaluable for studying natural and synthetic molecular catalysts of redox reactions that are of interest in the context of energy: activation of small molecules, solar fuels production, etc. ${ }^{1,2,3}$ With the catalyst either attached to the electrode or free to diffuse in solution, the current response to changing the electrochemical driving force can inform on the properties of the catalyst. Most commonly, the response is used to measure turnover frequencies (TOF) and catalytic potentials whose values can benchmark the performance of the catalyst. Beyond this, the response can be used to learn about the sequence of events in the catalytic cycle. Understanding the voltammetric response in the context of sound kinetic models is also a requirement for improving the design and performance of each catalyst.

Since most synthetic molecular catalysts function in only one direction of the redox reaction (e.g. $\mathrm{O}_{2}, \mathrm{H}^{+}$or $\mathrm{CO}_{2}$ reduction), it makes sense that most previous kinetic models of electrocatalysis have aimed at describing unidirectional catalysis, and therefore included unidirectional (irreversible) chemical steps. Only recently have catalysts been synthesized that can work in both directions, to oxidize or produce $\mathrm{H}_{2}$ depending on the applied electrode potential, ${ }^{4}$ which gives increased urgency to the study of the corresponding voltammetric responses. We do this here, by focusing on bidirectional electrocatalysis by diffusive or immobilized synthetic catalysts or enzymes.

In this work we emphasize the distinction between "bidirectional catalysis" and "reversible catalysis" to characterize the catalytic voltammetric signals (e.g. those obtained with the electrocatalysts of $\mathrm{H}_{2}$ oxidation and/or production). There is no consensus in the literature regarding the use of these words; here we employ the definitions used by Armstrong and Hirst. $^{5}$

In this paper "bidirectional" implies that the catalysts can catalyse the reaction in both directions (figure 1B and $\mathrm{C}$ ), as opposed to only one-way (figure $1 \mathrm{~A}$ ). We call "catalytic preference" 4 the ratio of the maximal currents measured at high driving force in both directions (in the enzyme electrochemistry field, and particularly regarding hydrogenases, the expression "catalytic bias" has been used ${ }^{6-15}$ ). Since these two rates are measured under different experimental conditions, their ratio is not the equilibrium constant of the reaction; it is not set by the thermodynamics of the reaction, but depends on the catalyst's properties instead. 


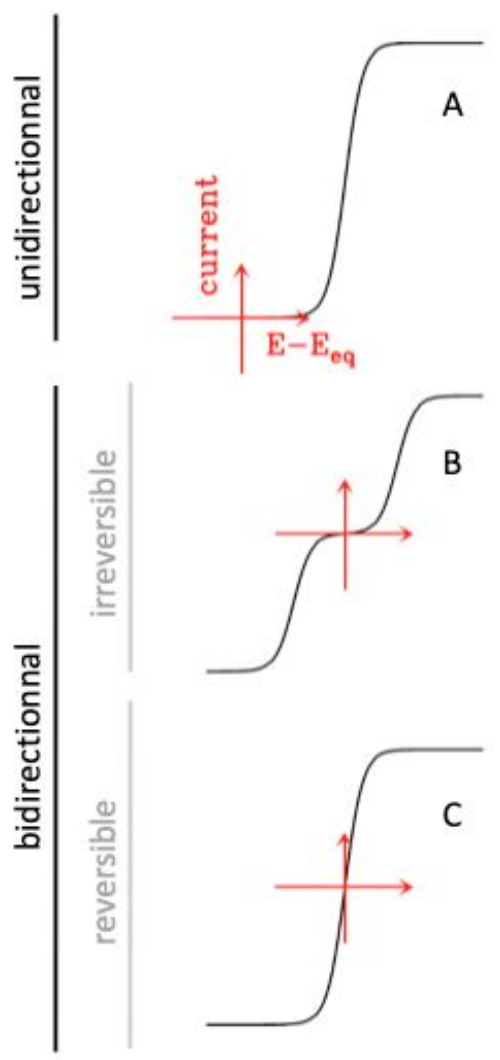

Figure 1.

Catalytic waveshapes for unidirectional $(A)$ and bidirectional catalysis ( $B$ and $C$ ), the latter either irreversible $(\mathrm{B})$ or reversible (C). For bidirectional catalysis, we chose to plot waves that show no catalytic preference for one particular direction of the reaction (the magnitude of the oxidative and reductive currents are the same). The current is zero at the equilibrium potential $\left.\left(E_{E q}\right)\right)^{16}$

While there are many distinct uses of the term "reversible", here we use the terms "reversible" and "irreversible" to describe the catalytic response at low overpotential, ${ }^{16}$ near the equilibrium (Nernst) potential. The adjective "reversible" describes the situation where a significant catalytic rate (current) is observed in either direction even for a small departure from the equilibrium potential. The reversible situation is opposed to the case where a large driving force (a large overpotential) is required to trigger catalysis. Figure $1 \mathrm{C}$ shows a catalytic wave that we describe as bidirectional and reversible, whereas panel B illustrates bidirectional and irreversible catalysis. Clearly, all reversible catalysts must be bidirectional, but not all irreversible catalysts are. However, the concurrent study of both irreversible and bidirectional catalysts can aid in understanding the details of the catalytic mechanism and what attributes should be designed to modify an irreversible catalyst to a reversible one.

Reversibility is a desirable property in the context of energy conversion, because it means that significant energy conversion rates can be obtained even at very low driving force, minimizing energy use. In the case of redox enzymes that function bidirectionally and reversibly and can be wired to an electrode, the measurement of the open circuit potential is a direct read-out of the Nernst potential of the corresponding couple, as indeed previously observed with enzymes that catalyze the conversions between $\mathrm{H}^{+}$and $\mathrm{H}_{2},{ }^{17}$ but also succinate/fumarate, ${ }^{18} \mathrm{CO}_{2} / \mathrm{CO},{ }^{19} \mathrm{CO}_{2} /$ formate $,{ }^{20} \mathrm{NADH} / \mathrm{NAD}^{+},{ }^{21}$ tetrathionate/thiosulfate,${ }^{22}$ etc. 
Regarding homogeneous non-electrochemical enzyme kinetics, uni- versus bidirectionality is usually discussed in the context of the Haldane equation, ${ }^{23,24}$ which, for a transformation between substrate and product, establishes the relation between the forward and backward maximal rates ( $v_{\max }^{f}$ and $v_{\max }^{b}$ respectively, extrapolated to infinite concentration of substrate or product, respectively), the substrate and product Michaelis constants ( $K_{M}^{S}$ and $\left.K_{M}^{P}\right)$ and the equilibrium constant: $K_{\mathrm{eq}}=[\mathrm{P}]_{\mathrm{eq}} /[\mathrm{S}]_{\mathrm{eq}}=v_{\max }^{f} K_{M}^{P} / v_{\max }^{b} K_{M}^{S}$. The concept of reversibility (having significant rate at low thermodynamic driving force) is not discussed in homogenous kinetics. This is unlike electrochemistry experiments, because the driving force is easily tuned by changing the electrode potential, while the kinetics is simultaneously measured as a catalytic current (figure 1).

Here we shall consider various mechanisms of bidirectional catalysis. We denote the models by a sequence of "E" and "C" symbols, which indicate redox and chemical steps, respectively, and their order in the catalytic cycle. All electrochemical steps are assumed to be reversible. The subscripts "i" or " $r$ " indicate the (ir)reversibility of the chemical step. For each mechanism, we shall compare two situations: the catalyst being either immobilized on an electrode or in solution; in the latter case, catalysis occurs in a diffusion layer that extends in the solution, away from the electrode. The former case is relevant to studies of both enzymes and small catalysts attached to electrodes; ${ }^{25-28}$ the latter is probably pertinent only for small synthetic catalysts, since a large enzyme diffusing slowly to/from the electrode would give a small catalytic current (unless soluble redox mediators are used, but we do not address this situation here).

In the context of solar fuels, all reactions of interest are multi-electron redox processes $\left(\mathrm{H}_{2}\right.$ oxidation, $\mathrm{CO}_{2}$ reduction, water oxidation, $\mathrm{N}_{2}$ reduction, etc.), and yet, most of the available electrochemical models of catalysis consider a single one-electron step. The one-electron $E_{r}$ model has been considered by Savéant for a solubilized catalyst ${ }^{29}$ and by Armstrong for an adsorbed catalyst. ${ }^{30}$ These two models cannot be used to discuss the difference between directionality and reversibility since all bidirectional waves calculated from one-electron models predict that the signal is a one-electron sigmoid (potentially distorted by slow interfacial electron transfer), as shown in figure $3 \mathrm{~A}$. In other words, these models do not have the minimal complexity that is required to predict waves such as those in figure $1 \mathrm{~B}$. Two-electron models for unidirectional catalysts in solution have only recently been described ${ }^{31}$ and discussed. ${ }^{1,32}$ On our side, we have described various two-electron models of bidirectional catalysis $\left(E E C_{r}, E E C_{r} C_{r}\right.$ and $\left.E C_{r} E C_{r}\right)$ for an adsorbed catalyst and we have examined the effect of internal redox relays ${ }^{33}$. We have applied this theory to enzymes, but data are now also available for adsorbed synthetic catalysts. ${ }^{25-28}$ Here we extend our work to the case where the bidirectional catalyst diffuses in solution.

We shall describe which catalytic signals are allowed by distinct mechanisms $\left(E E C_{r}, E E C_{r} C_{r}\right.$, $E E C_{r} C_{r} C_{r}$ and $E C_{r} E C_{r}$ ), and how the shapes of the signals depend on the parameters of the model. Our goal is two-fold: to establish whether or not a particular catalytic signature is a fingerprint of a certain mechanism, and to identify the kinetic determinants of bidirectionality and reversibility. Hence we evaluate which conditions on the parameters of each kinetic model must be met for the signal to be bidirectional and/or reversible, and we show that 
there are intrinsic reasons, related to the properties of the catalytic intermediates and the rates of the elementary steps, why the catalyst may behave reversibly or irreversibly, irrespective of the rate of interfacial electron transfer. We hope that this will enable using electrochemistry to decipher the mechanisms of bidirectional electrocatalysis, and for guiding the design of reversible solar-fuel catalysts.

\section{Results}

\section{Mechanisms}

Figure 2 shows the mechanisms that we discuss in this paper. $E_{1}^{0}$ and $E_{2}^{0}$ are the two one-electron non-catalytic potentials of the catalyst. The catalytic cycles are closed by a series of reversible chemical steps. The rate constants with positive subscripts ( $k_{1}$ and $\left.k_{2}\right)$ are in the direction of reductive catalysis $\left(\mathrm{H}_{2}\right.$ production). The catalytic cycle must include protonation steps, which may either be fast and coupled to reduction (in which case the corresponding $E^{0}$ depends on $\mathrm{pH}$ ) or slow and treated as one of the chemical steps. The same is true for substrate binding/release.

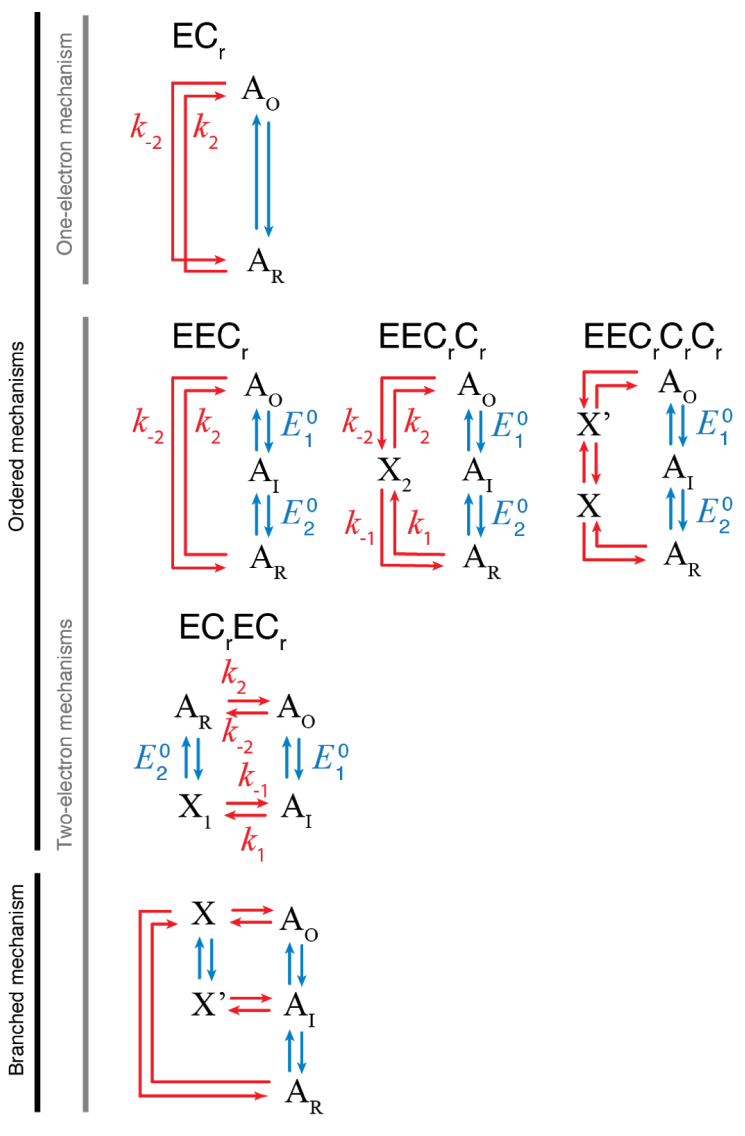

Figure 2

The ordered mechanisms discussed here $\left(E C_{r}, E E C_{r}\right.$, $E E C_{r} C_{r}, E E C_{r} C_{r} C_{r}, E C_{r} E C_{r}$ ) and an example of branched mechanism. $A$ is the catalyst (or the active site of the enzyme), and subscripts $O$, $\mathrm{I}$ and $\mathrm{R}$ denote redox states that are oxidized, intermediate (singly reduced) and fully reduced, respectively. $X_{i}$ denotes a catalytic intermediate. Blue arrows indicate redox steps, and red arrows chemical steps, with positive subscripts for the direction of reductive catalysis (e.g. $\mathrm{H}_{2}$ production). We shall note $K_{i}=k_{i} / k_{-i}$ the equilibrium constants of the chemical step $i$. 


\section{Assumptions}

The calculations of these steady-state catalytic signals are much easier for the situation where the catalyst is adsorbed than when it diffuses. In the former situation, the calculation involves the resolution of a system of linear equations with potential-dependent coefficients (these equations describe the evolution of each of the catalytic intermediates). ${ }^{33}$ In contrast, if catalysis occurs in solution, one must consider the concentration profiles of all catalytic intermediates and the boundary conditions on the electrode (we show these derivations in the $\mathrm{SI})$.

While we try to limit our assumptions, some are necessary to make the derivation tractable. We shall consider only direct electron transfer between the electrode and the catalyst, and the situation where this electron transfer is fast and reversible (i.e. Nernstian); the validity of the latter assumption is discussed below. When the catalyst is in solution, we assume that all catalytic intermediates diffuse with the same diffusion coefficient $D$; we also focus on so-called "canonical conditions", ${ }^{29}$ a particular limiting situation where the different forms of the catalyst reach a steady-state concentration profile by mutual compensation of catalysis and catalyst diffusion, and the substrate concentration is large enough that depletion near the electrode surface is negligible. The equivalent limiting situation is achieved with adsorbed catalysts when the scan rate is small and the electrode rotation rate and bulk substrate concentrations are high. In this limit, in both cases, the catalytic response is S-shaped (like all voltammograms in figure 1 ) and independent of scan rate and scan direction; it reveals the intrinsic properties of the catalyst.

Regarding the theory developed in this paper, we shall consider situations where the catalytic cycle is the same irrespective of the driving force (in particular, the mechanism is the same in the two directions of the reaction, only the order of the steps is reversed).

Enzymologists would describe these mechanisms as "sequential" (since all the substrates, protons and electrons, bind the enzyme before the product of the reaction is formed) and "ordered" (the substrates combine with the enzyme and the product dissociates in an obligatory order). ${ }^{23}$ However, regarding both redox enzymes ${ }^{37,38}$ and synthetic catalysts, ${ }^{39-47}$ there have been suggestions that changing the electrode potential or the concentration of substrate may force the catalytic cycle to take distinct routes, and therefore the assumption that the mechanism is ordered requires assessment on a case-by-case basis. Enzymologists call "sequential and random" ${ }^{23}$ the mechanisms that imply no obligatory order of combination of the substrate; we suggest using the term "branched" instead of "random", because such a mechanism may occur if there is a branch point along the catalytic reaction pathway, where alternative transformations compete and determine the TOF. The bottom scheme in figure 2 illustrates a case where one of the two competing reactions is a redox step, in which case changing the rate of the electron transfer step by changing the potential should force the system to either take or avoid the route along the redox step. Similarly, changing the rate of the chemical steps (e.g. by changing the strength of the acid) can also determine which pathway is operational. For example, nickel catalysts for proton reduction and hydrogen oxidation have been noted to switch mechanisms if a strong acid vs. a weak acid is used, or conversely if a strong base vs. a weak base is used. ${ }^{40-45}$ This behaviour has 
also been observed for a NiRu dinuclear catalyst. ${ }^{46}$ Additionally, depending on the strength of acid used, cobaloxime catalysts can display three distinct catalytic waves spanning a $1 \mathrm{~V}$ difference in catalytic potential. ${ }^{47}$

That the mechanism is ordered implies that the oxidation and reduction follow the same catalytic pathway, only the steps are reversed. A branched mechanism can switch between paths as the experimental conditions changes: distinct pathways may coexist at a given potential, and distinct mechanisms may dominate under distinct experimental conditions. It may be, for example, that proton reduction at very low potential follows an EECC mechanism, while $\mathrm{H}_{2}$ oxidation at high potential follows the ECEC route. But "branched" does not necessarily mean that oxidative and reductive catalysis follow distinct routes, and in particular, it cannot happen that the mechanism switches from one path to another just upon crossing the equilibrium potential: the mechanism operating near the equilibrium potential for a reversible catalyst must be operational in both directions, and any change in mechanism must happen continuously as the experimental conditions are varied, going through conditions where the two mechanisms coexist.

\section{Regarding the assumption that interfacial electron transfer is fast}

Considering molecular electrocatalysis, the Nernstian limit describes the situation where the rate of interfacial electron transfer between the electrode and the catalyst is so fast that the redox transformations remain at equilibrium; the resulting rate equation combines terms that are either independent of potential or proportional to $\exp (n F E / R T)$ ( $n=1$ or $n=2, E$ is the electrode potential).

For adsorbed catalysts, the validity of the Nernstian approximation requires that the rate of interfacial electron transfer (the prefactor $k_{0}$ in the Butler Volmer equation, in units of $\mathrm{s}^{-1}$ for adsorbed species) be much larger than the turnover frequency $k_{\text {cat }}$. Regarding adsorbed enzymes, this approximation fails in most cases, and the slow rate of interfacial ET (and sometimes a distribution thereof) is explicitly taken into account to describe the catalytic wave shape. ${ }^{48}$

In contrast, regarding analytical models of the electrochemical response of catalysts in solution, only the Nernstian limit is ever considered. ${ }^{3}$ But note that this assumption is valid only on the condition that $k_{0} \gg \sqrt{D k_{\text {cat }}}$ where $D$ is the diffusion coefficient of the catalyst in solution. Very few values of $k_{0}$ for inorganic catalysts have been reported in the literature; those reported are lower than $1 \mathrm{~cm} / \mathrm{s}$ and often in the range $10^{-4}-10^{-3} \mathrm{~cm} / \mathrm{s}^{49,50,51}$ Additionally, the microstructure and polishing history of glassy carbon electrodes has been noted to change the $k_{0}$ value of a molecular complex by orders of magnitude. ${ }^{52,53}$ Assuming $k_{0}=10^{-3} \mathrm{~cm} / \mathrm{s}$ and $D=10^{-6} \mathrm{~cm}^{2} / \mathrm{s}$, the validity of the Nernstian approximation requires $k_{\text {cat }}<1 \mathrm{~s}^{-1}$. Therefore, we expect that this approximation should fail as more and more active catalysts are designed in the future. Whether or not this condition is met can be assessed on a case-by-case basis by examining the shapes of the voltammograms, since interfacial ET limitations broaden the signal. 
With a one-electron model, the only way to obtain irreversible bidirectional catalysis (figure $1 \mathrm{~B}$ ) is when very slow interfacial electron transfer flattens the signal. Indeed in ref ${ }^{5}$ for example, irreversibility is said to result from slow interfacial electron transfer. But by including a second redox step in our models of catalysis, we show here and in ref ${ }^{33}$ that bidirectional, irreversible catalysis can also be observed in the Nernstian limit (figure 3B), which is our focus in this paper.

\section{Features that are common to all two-electron mechanisms considered here}

Under the assumptions described above, whether the catalyst is adsorbed or in solution makes no difference to the kind of catalytic signals that are predicted: they are all S-shaped, consisting of either a sharp (two-electron) wave (figure $3 \mathrm{C}$ ) or two broader (one-electron) waves (figure $3 \mathrm{~B}$ ). Equation 1 below is the generic equation that gives the catalytic current response for any two-electron ordered mechanisms:

$$
i(E)=\frac{i_{\mathrm{lim}}^{\mathrm{ox}} e_{\mathrm{cat}}^{\mathrm{ox}, \mathrm{n}=1} e_{\mathrm{cat}}^{\mathrm{red}, \mathrm{n}=1}-i_{\mathrm{lim}}^{\mathrm{red}}}{1+e_{\mathrm{cat}}^{\mathrm{red}, \mathrm{n}=1}\left(1+e_{\mathrm{cat}}^{\mathrm{ox}, \mathrm{n}=1}\right)}
$$

were we note $e_{\mathrm{cat}}^{\mathrm{ox}, \mathrm{n}=1}=\exp ^{F\left(E-E_{\mathrm{cat}}^{\mathrm{ox}, \mathrm{n}=1}\right) / R T}$ and $e_{\mathrm{cat}}^{\mathrm{red}, \mathrm{n}=1}=\exp ^{F\left(E-E_{\mathrm{cat}}^{\mathrm{red}, \mathrm{n}=1}\right) / R T}$.

We demonstrate in the SI section S2 that eq. 1 is valid irrespective of the number of chemical steps in the cycle and irrespective of whether the catalyst is adsorbed or diffuses, provided the mechanism is ordered. This implies that the experimental confirmation of equations 1 (or eq. 2 below) can be considered a confirmation that the mechanism is ordered at least over the entire potential range used to check eqs 1 or 2 .

If the mechanism is branched but the alternate pathways are fast on the time scale of turnover, the mechanism should still be considered ordered from the point of view of the rate equation, and eq. 1 is still valid. ${ }^{6,18,37,54}$

Figure 3 defines the meaning of the parameters that characterize the shape and magnitude of the wave. The plateau currents in either direction are $i_{\lim }^{\mathrm{ox}}$ and $i_{\mathrm{lim}}^{\mathrm{red}}$, and their ratio $i_{\mathrm{lim}}^{\mathrm{ox}} / i_{\mathrm{lim}}^{\mathrm{red}}$ defines the so-called "catalytic preference"; we also define, as in ref ${ }^{33}$, two catalytic potentials, $E_{\text {cat }}^{n=1, \text { ox }}, E_{\text {cat }}^{n=1 \text {,red }}$, which are the mid-point potentials of the two one-electron waves seen in figure 3B. These catalytic potentials may differ from the two redox potentials of the catalysts, $E_{1}^{0}$ and $E_{2}^{0}$, determined in the absence of catalysis: such "kinetic shifts" are expected in most cases, as discussed in detail herein. These parameters can be measured by fitting the experimental signal, ${ }^{48}$ and their expressions can also be related to the parameters in the models in figure 2, as indicated in Table 1, which we discuss below.

We compile in Table 1 the relations between the parameters that define the waveshapes ( $E_{\text {cat }}$ 's and $i_{\text {lim }}$ 's) and the parameters (rate constants and redox potentials) in each of the schemes in figure 2. 
We also tabulate the equations of the TOF ${ }^{\max }$ in each direction, which we define as the turnover frequency in the limit of very high overpotential, where the redox steps become very fast and irreversible. The TOF ${ }^{\max }$ values are the same irrespective of whether the system is adsorbed or free to diffuse.

The magnitude of the current in either direction $\left(i_{\lim }^{\text {ox }}, i_{\lim }^{\text {red }}\right)$ is proportional to either the electroactive coverage $\Gamma$ (for an adsorbed catalyst) or the product $C \times \sqrt{D}$ (where $C$ is the catalyst concentration and $D$ its diffusion coefficient). In contrast, the shape of the signal (determined by $E_{\mathrm{cat}}^{n=1, \mathrm{ox}}, E_{\mathrm{cat}}^{n=1, \text { red }}, i_{\mathrm{lim}}^{\text {ox }} / i_{\lim }^{\text {red }}$ ) depends only on the two redox potentials of the catalyst and the rate constants of the chemical steps in the model, not on the coverage or concentration of catalyst.

The expressions of the limiting currents and catalytic potentials in Table 1 are different and much simpler in the adsorbed case than when the catalyst diffuses. The limiting currents for the adsorbed case are proportional to TOF ${ }^{\max }$ irrespective of whether the mechanism is unior bidirectional. But when the catalyst diffuses, the limiting current in a given (forward) direction depends on all rate constants and on the size of the catalyst's diffusion layer, which is affected by the rate constants of the forward and backward chemical steps. Thus increasing the rates of the backward chemical steps may decrease the forward limiting current. The well-known relation between the limiting current for the diffusive case and the square root of TOF ${ }^{\text {max }}, i_{\text {lim }}=n F A C \sqrt{\mathrm{TOF}^{\mathrm{max}} D}$, which is true for unidirectional mechanisms, does not hold for any bidirectional mechanism (not even $\mathrm{EC}_{\mathrm{r}}{ }^{29}$ ); using this incorrect equation leads to underestimations in TOF ${ }^{\max }$.

In all cases, the fact that the current is zero when the electrode potential equates the Nernst potential of the substrate/product couple ( $\left.E_{\text {eq }}\right)$ imposes a relation between the parameters in eq 1: the catalytic preference is related to the difference between $E_{\text {eq }}$ and the two-electron catalytic potential $E_{\text {cat }}^{n=2}$ by the relation :

$$
\frac{i_{\mathrm{lim}}^{\text {ox }}}{i_{\mathrm{lim}}^{\text {red }}}=\exp \left(\frac{2 F}{R T}\left(E_{\mathrm{cat}}^{n=2}-E_{\mathrm{eq}}\right)\right)
$$

Importantly, $E_{\text {eq and }} E_{\text {cat }}^{n=2}=\left(E_{\text {cat }}^{n=1, \text { ox }}+E_{\text {cat }}^{n=1, \text { red }}\right) / 2$ both are "two-electron" potentials. Regarding complex enzymes, where one or several redox sites mediate long range electron transfer between the active site and the surface of the enzyme, there are no grounds for replacing $E_{\text {cat }}^{n=2}$ in eq 2 with the one-electron redox potential of a one-electron "control site" where electrons enter or leave the enzyme, as suggested by others. ${ }^{30}$ 

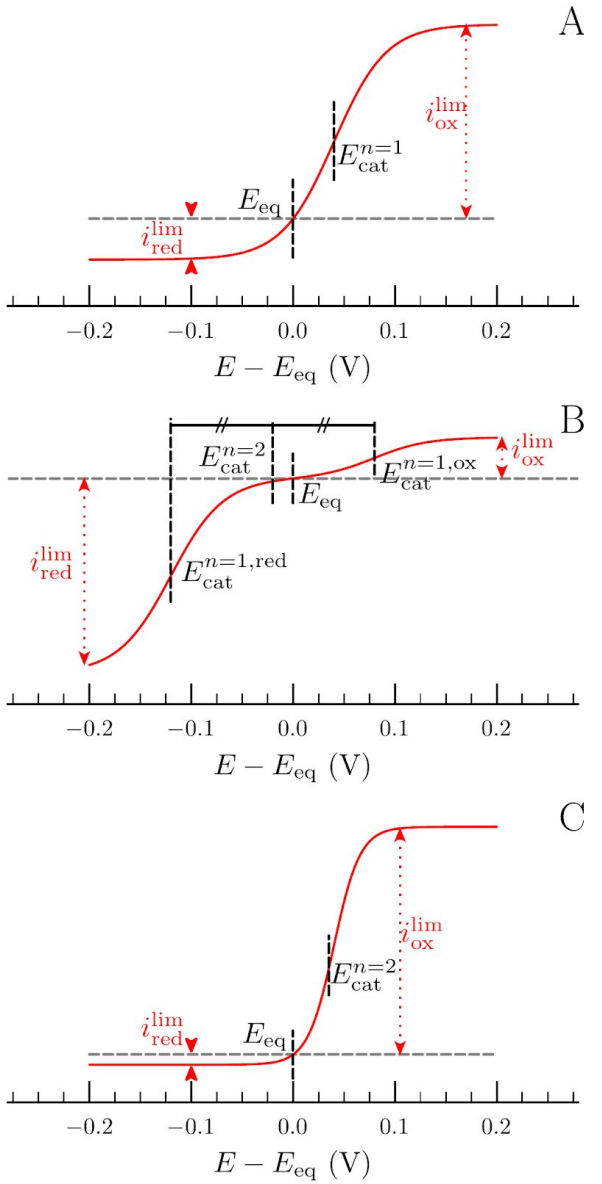

Figure 3

The typical, "Nernstian", bidirectional electrochemical responses, calculated from the ordered models in figure 2, and definition of the phenomenological parameters (catalytic potentials and limiting currents) that characterize the shape and position of each signal.

A: A bidirectional and reversible catalytic wave, as predicted by Savéant ${ }^{29}$ using a one-electron models of catalysis $\left(E_{\mathrm{r}}\right)$. B: A bidirectional and irreversible, two-electron catalytic signal C: A bidirectional and reversible, two-electron catalytic signal (sharper than the one-electron sigmoids in panels $A$ and $B$ ). The catalytic potentials are the positions of the inflection points of the catalytic waves. For any signal calculated from two-electron models (B and $\mathrm{C}$ ), we define $E_{\text {cat }}^{n=2}=\left(E_{\text {cat }}^{n=1, \text { ox }}+E_{\text {cat }}^{n=1, \text { red }}\right) / 2$ as in ref ${ }^{33}$.

Equation 2, which is the equivalent for electrocatalysis to the Haldane equation in homogeneous enzyme kinetics, ${ }^{23,24}$ defines the catalytic preference of the bidirectional catalyst. A positive difference between $E_{\text {cat }}^{n=2}$ and $E_{\text {eq implies that the oxidative limiting }}$ current is larger than the reductive limiting current. The catalytic preference increases ten-fold for each $30 \mathrm{mV}$ additional separation between $E_{\text {cat }}^{n=2}$ and $E_{\text {eq. }}$.

In contrast, the reversibility of the wave depends on the difference between $E_{\text {cat }}^{n=1, \mathrm{ox}}$ and $E_{\text {cat }}^{n=1 \text {,red }}$, as shown in figure 3B and C. A positive value of $E_{\text {cat }}^{n=1, \text { ox }}-E_{\text {cat }}^{n=1 \text {,red }}$ corresponds to irreversible catalysis (figure $3 \mathrm{~B}$ ), whereas the potentials being crossed over ( $E_{\text {cat }}^{n=1, \text { ox }}<E_{\text {cat }}^{n=1 \text {,red }}$ ) gives a reversible, two-electron wave centered on $E_{\text {cat }}^{n=2}=\left(E_{\text {cat }}^{n=1, \text { ox }}+E_{\text {cat }}^{n=1, \text { red }}\right) / 2$ (figure 3C).

The bottom part of Table 1 shows that the catalytic potentials depend on the non-catalytic redox potentials $E_{1}^{0}$ and $E_{2}^{0}$, but depart from these values in all cases except the simple $E C_{r}$ mechanism. This means the reversibility of the catalyst depends on the two one-electron potentials of the catalyst in all cases, and also on the values of the rate constants of the chemical steps if the model is more complex than $E_{E C}$. 


\section{The $E C_{r}$ mechanism}

This simplest mechanism in figure 2 includes two electron transfers ("EE") and takes into account the combination of all other elementary steps within the cycle as a single, composite chemical step, " $\mathrm{C}_{\mathrm{r}}$ ", with pseudo-1st order rate constants $k_{2}$ in the direction of $\mathrm{H}_{2}$ evolution, $k_{-2}$ for $\mathrm{H}_{2}$ oxidation.

Regarding the magnitude of the corresponding catalytic signal, the limiting reductive current simply depends on $k_{2}$ in the case of an adsorbed catalyst (eq 1 in table 1), whereas it is proportional to $k_{2} \sqrt{D /\left(k_{2}+k_{-2}\right)}$ if the catalyst diffuses (eq 3 in table 1 ). In the latter case, the reason $i_{\text {lim }}^{\text {red }}$ depends not only on $k_{2}$ but also on $k_{-2}$ is that, if all other parameters remain the same, increasing $k_{-2}$ decreases the size of the reaction-diffusion layer near the electrode where catalysis occurs, and therefore decreases the reductive current.

However, the equation of the catalytic preference is very simple, $i_{\lim }^{\mathrm{red}} / i_{\mathrm{lim}}^{\mathrm{ox}}=k_{2} / k_{-2}$, irrespective of whether the catalyst is adsorbed or solubilized. This bias merely equates the equilibrium constant of the composite chemical step.

Also irrespective of whether the catalyst is adsorbed or diffuses, the two values of $E_{\text {cat }}^{n=1}$ equate the two one-electron potentials of the catalyst $E_{1}^{0}$ and $E_{2}^{0}$ (eqs $12,13,14,15$ in Table 1), as shown in figure $4 \mathrm{~A}$. The $\mathrm{EEC}_{\mathrm{r}}$ mechanism is the only reversible mechanism where we predict no kinetic shift between $E_{\text {cat }}^{n=1}$ and $E^{0}$. 


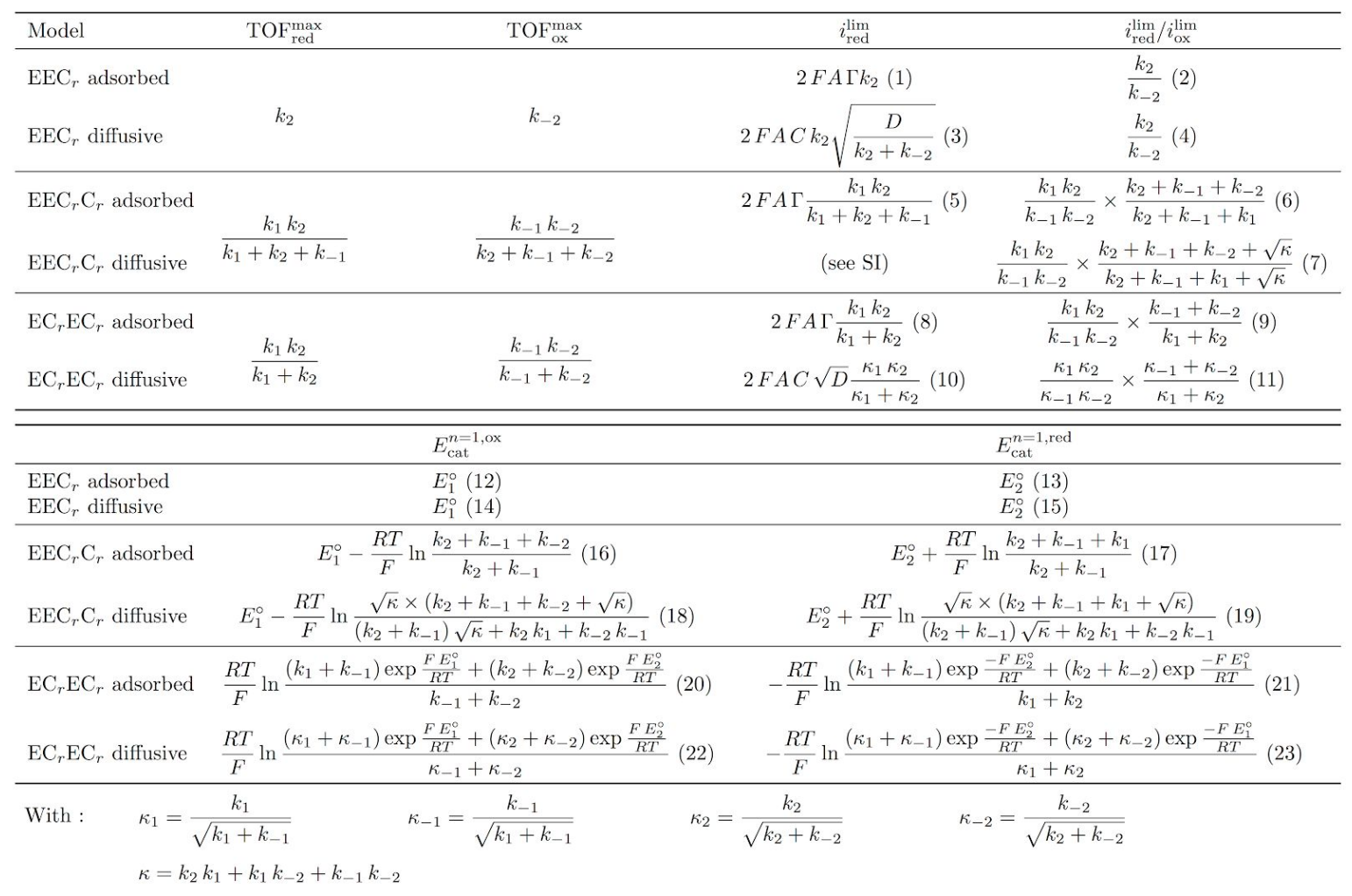

Table1: The TOF ${ }^{\max }$ and electrochemical response (the limiting reductive currents, the catalytic preference, and the two catalytic potentials defined in eq 1 and figure 3 ) for three of the mechanisms in figure 2 and the two configurations (adsorbed or diffusing catalyst). The rate constants and redox potentials are defined in figure 2. All equations are derived in ref ${ }^{33}$ for the "adsorbed case" and in SI for the "diffusive case". 
A

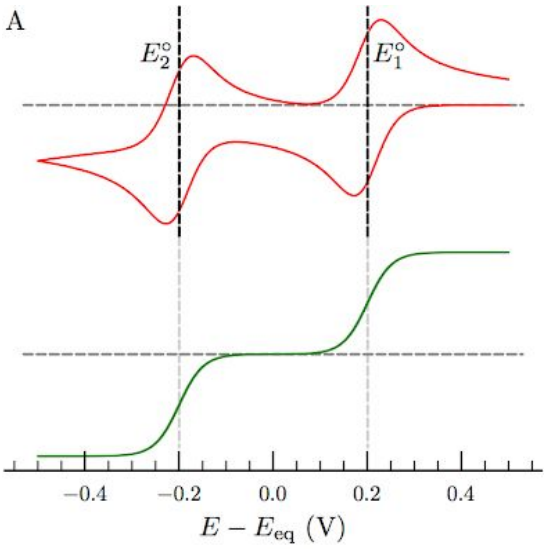

B

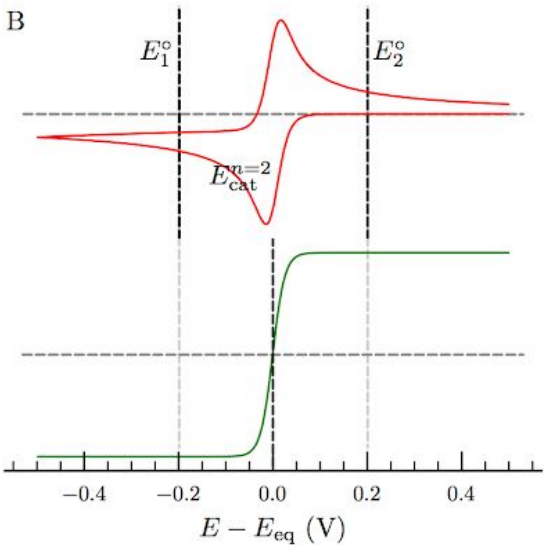

C

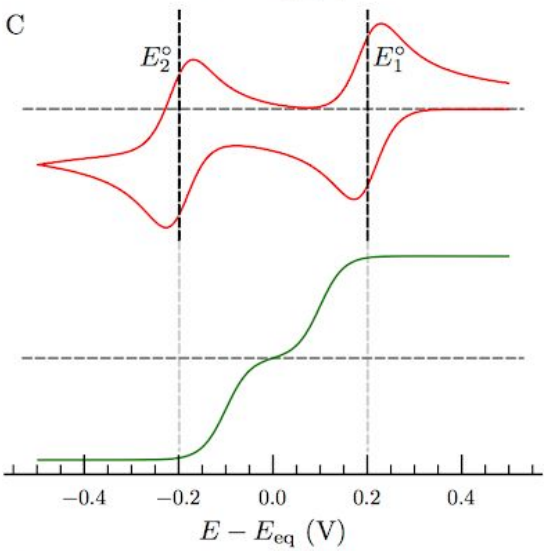

$\mathrm{D}$

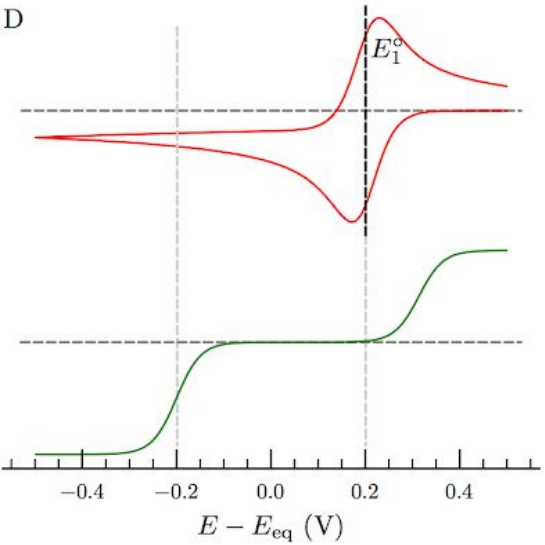

Figure 4

Current/potential responses illustrating which relations can be observed between non catalytic (red) and catalytic (green) electrochemical signals.

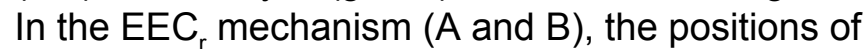
the two catalytic waves match the non-catalytic potentials and reversibility results from the two potentials being crossed over (B). In the $E_{E} C_{r} C_{r}$ mechanism $(C)$, the two catalytic waves can only be closer to one another than the non catalytic potentials. In the $E_{r_{r}} E_{r}$ mechanism (D), a single non catalytic reaction may be seen, and the value of the catalytic potentials is not simply related to the corresponding non-catalytic potential(s). Table S1 shows the values of the parameters used to calculate these signals, chosen so that the catalytic preference equals one. 
This implies that the reversibility of the catalytic signal is simply related to the stability of the singly reduced intermediate, which can in principle be measured by recording a cyclic voltammogram under non-catalytic conditions (e.g. for a $\mathrm{H}^{+} / \mathrm{H}_{2}$ catalyst, in the absence of acid and $\mathrm{H}_{2}$ ). If the singly reduced state is stable over a large potential range $\left(E_{1}^{0}>E_{2}^{0}\right)$ then the catalytic signal is irreversible as shown in figure $3 \mathrm{~B}$. If the reduction of the catalyst is a two-electron cooperative process $\left(E_{1}^{0}<E_{2}^{0}\right)$ then the catalytic signal is sharp and reversible (figure $3 \mathrm{C}$ ).

\section{$E C_{r} C_{r}$}

Regarding the case where the catalyst is adsorbed and the $E_{E} C_{r} C_{r}$ mechanism applies, the equations that give the magnitude of the limiting current in either direction (eqs 5 in table 1 and S31 in SI) are more complex than for the EEC mechanism. For the adsorbed case, the expression of the current includes a penalty by one of the backward rate constants $\left(k_{-1}\right.$ at the denominator of eq 5 in Table 1). In the diffusive case, the equations of the limiting currents are very complex and are shown only in the SI (section S5).

The expressions of the catalytic preferences (eqs. 6 and 7 in Table 1) are also non-trivial. In both cases (diffusive and adsorbed catalysts), the ratio of limiting currents does not equate the product of the equilibrium constants of the two chemical steps $\left(K_{1} K_{2}\right.$, we note $\left.K_{i}=k_{-i} / k_{i}\right)$, unless $k_{-2}$ and $k_{1}$ are small $\left(k_{-2}<k_{2}+k_{-1}\right.$ and $k_{1}<k_{2}+k_{-1}$, which occurs when the intermediate $\mathrm{X}_{2}$ in figure 2 is very unstable).

The catalytic potentials depart from the values of $E_{1}^{0}$ and $E_{2}^{0}$. Equations $16,17,18, \& 19$ in Table 1 clearly show that in both cases (diffusive and adsorbed catalysts), $E_{\text {cat }}^{n=1, \text { ox }}$ is lower than $E_{1}^{0}$, and $E_{\text {cat }}^{n=1 \text {,red }}$ is greater than $E_{2}^{0}$ : each wave is kinetically shifted from the corresponding values of $E^{0}$ in the direction of lesser driving force. This has a direct and simple consequence: the two chemical steps draw the two catalytic potentials nearer, and make the catalytic wave more reversible than is expected based on the thermodynamic stability of the singly reduced active site measured in the absence of catalysis. The conclusion that the two catalytic potentials are necessarily more crossed over than the non-catalytic potentials if the $E E C_{r} C_{r}$ mechanism applies is illustrated in figure $3 \mathrm{C}$.

\section{$E^{E} C_{r} C_{r} C_{r}$}

We examined the robustness of the above conclusion by examining the voltammetry of an even more complex $E E C_{r} C_{r} C_{r}$ mechanism, and we found that considering a third chemical step does not change the conclusion we reached about the $E_{E C_{r}} C_{r}$ model, according to which the two catalytic potentials are more crossed over than the non-catalytic potentials.

To demonstrate this finding, instead of solving the equations for the values of $E_{\text {cat }}$ and the limiting currents, we ran a series of thousands of simulations, choosing random values of the rate constants of the chemical steps and calculating the corresponding waves, to observe 
whether some parameters resulted in the waves being pushed apart from the non-catalytic potentials; we did not find any (see SI section S6).

\section{$E_{r} E_{r}$}

Regarding the limiting currents, the catalytic preference and the catalytic potentials, the equations for the solubilized case are more complex than the equations for the adsorbed case (see the $\mathrm{SI}$ ), but can be simplified by replacing the true rate constants $k_{1}, k_{2}, k_{-1}, k_{-2}$ with effective rate constants $\kappa_{i}$, defined in Table 1 . This simplification being taken into account, the discussion of the solubilized and adsorbed cases is the same.

The expressions of the limiting currents for the $E_{\mathrm{r}_{\mathrm{r}}} \mathrm{C}_{\mathrm{r}}$ mechanism are rather simple (eqs 8 and 10 in Table 1), because the intermediate E step "uncouples" the two chemical steps: at very high or very low potential, the very fast electron transfers prevent backward reactions, making the preceding chemical step effectively irreversible. Each limiting current is therefore proportional to the sum of the reciprocal of the rates of the two steps in the corresponding direction (e.g. $1 / i_{\lim }^{\text {red }} \propto 1 / k_{1}+1 / k_{2}$ for the adsorbed case).

This implies that the catalytic preference is also given by the ratio of the slowest rate constants in each direction. It may be that the same chemical reaction is the slowest step in each direction, in which case the catalytic preference is the equilibrium constant of this reaction (e.g. $k_{1} / k_{-1}$ ). Or it may be that the two chemical reactions each are the slowest step in one of the two directions, in which case the preference is a ratio of two rate constants that do not correspond to the same "C" reaction (e.g. $k_{1} / k_{-2}$ ) and it does not equate to an equilibrium constant. Each of these two extreme cases has actually been observed in our previous work on hydrogenase..$^{7,8}$

A major difference with respect to the previous mechanisms is that the second redox process in the sequence "EC $E_{r} C_{r}$ " can be detected in the absence of catalysis only on the condition that the second $\mathrm{C}_{\mathrm{r}}$ reaction, which closes the catalytic cycle, can be prevented. If we consider $\mathrm{a}_{2}$ production catalyst, both C's are probably protonation steps, and it may be difficult to find conditions where the two E's show up in the non-catalytic voltammetry. If both $\mathrm{C}_{\mathrm{r}}$ steps are prevented, e.g. by having no protons in the solvent, the $1^{\text {st }}$ "E" can be detected, but no other detectable redox process can correspond to the second $E$ in "EC $\mathrm{r}_{\mathrm{r}} \mathrm{C}_{\mathrm{r}}$ ". For this reason, there can be no simple relation between the values of the two catalytic potentials and the value of the only relevant catalyst's potential that is seen in the absence of any " $\mathrm{C}_{r}$ " reaction. Each of the two catalytic potentials depends on the four rate constants and on the two non-catalytic potentials (eqs $20,21,22 \& 23$ in Table 1), only one of which can be measured under non catalytic conditions. This is illustrated in figure 3D.

\section{Discussion}

A significant difference between our approach and that from other groups is that we consider mechanisms that are as general as possible: for example, we have predicted the magnitude 
and shape of the voltammograms without making any a priori assumptions about the difference between the values of $E_{1}^{0}$ and $E_{2}^{0}$ (allowing the two potentials to be crossed over or uncrossed) or the values of rate constants of the chemical steps in each model. A more common approach is to begin with stronger assumptions (meaning, simpler mechanisms, which depend on a minimal number of parameters) and to assume that they allow a meaningful interpretation of the data. Care should be taken that simple mechanistic scenarios may result in predictions that are not general. For example, catalytic mechanisms that include a single chemical step (e.g. $\mathrm{EC}_{i}, \mathrm{EEC}_{\mathrm{r}}$ etc.) predict that the catalytic potentials (the potentials where catalysis occurs) equate the non-catalytic redox potentials of the catalyst, whereas with more complex mechanisms (e.g. $E_{C_{i}} C_{i},{ }^{31} E_{E C_{r}} C_{r}$ etc.), the catalytic waves may deviate from the non-catalytic potentials. Hence the assumption that the catalytic wave is centered on the non-catalytic potential of the catalyst should not be made unless its validity is first ascertained. This caveat applies to the popular "foot of the wave analysis", $1,34,35$ which consists of extrapolating the plateau current from the onset of the sigmoidal catalytic unidirectional wave, assuming a certain value of the catalytic potential: mistaking the value of the catalytic potential yields an incorrect value of the plateau current. ${ }^{36}$ It is therefore useful in this paper to describe complex, diverse and realistic mechanistic scenarios such as those in figure 2 , to understand the range of possible behaviors, and to clarify which conclusions are robust and which are model-dependent.

\section{In addition to the equilibrium potential, two potentials matter, not one}

A major conclusion here is that to discuss the shape of the catalytic signals for ordered mechanisms, we needed to define and consider the values of two one-electron catalytic potentials (and/or their average value, a two-electron potential) (figure 3). Eq 2 shows that the catalytic preference (directionality) depends on the difference between the average two-electron catalytic potential and the equilibrium potential (the two-electron $\mathrm{H}^{+} / \mathrm{H}_{2} \mathrm{Nernst}$ potential), as discussed previously, see ref ${ }^{33}$ and Box 1 in ref ${ }^{55}$, whereas reversibility is determined by the difference between the two one-electron catalytic potentials (figure 3 ).

The reason this idea is largely absent from the literature ${ }^{33,55}$ is probably that unidirectional two-electron catalysis with diffusing catalysts was most often described in terms of one-electron models, where the second redox step is considered fast (hence not rate determining) in the potential window where catalysis is observed. This one-electron paradigm was naturally developed further in one-electron models of bidirectional catalysis; all these one-electron models predict that the catalytic wave should be a single, one-electron sigmoid (figure 1A) unless slow electron transfer broadens the signal. ${ }^{29,30}$ In his recent paper about bidirectional catalysis in solution, Savéant identifies the standard potential of the one-electron catalyst as the main determinant of directionality and reversibility (in this approach, the two cannot be distinguished). ${ }^{29}$

In summary, in the one-electron models of catalysis, ${ }^{29,30}$ bidirectionality and reversibility are one and the same, the whole shape of the wave is defined by a one-electron potential (either the catalyst or the redox center) and the catalytic preference is simply related to the difference between this potential and the equilibrium potential. The conclusions reached in 
this paper, based on the discussion of two-electron models of two-electron catalysis, are significantly different: bidirectionality and reversibility are distinct features of the signal, which are defined by two catalytic potentials plus the value of the equilibrium potential.

\section{Identifying the sequence of events in the catalytic cycle by examining how the position of the waves compares to the non-catalytic potentials}

Attempts to understand the catalytic mechanism of $\mathrm{H}_{2}$ oxidation and production catalysts have relied on the interpretation of electrochemical data. Two sequences of events are usually distinguished for proton and electron uptake in the catalytic cycle $\left(E_{\mathrm{r}} E \mathrm{C}_{\mathrm{r}}\right.$ and EEC $\left._{r} C_{r}\right)$.

We have shown that irrespective of the ordered catalytic mechanism in figure 2, the catalytic signal consists of either two one-electron sigmoids or one two-electron sigmoid (eq 1 and

figure 3 ), the positions of which are defined by two catalytic potentials, $E_{\text {cat }}^{n=1 \text {,ox }}$ and $E_{\text {cat }}^{n=1 \text {,red }}$ . The shape of a voltammogram alone cannot be used to determine which of the ordered mechanisms considered here is operational, but the comparison between the catalytic potentials (the mid points potentials of the catalytic waves) and non-catalytic potentials (the redox potentials determined in the absence of catalysis) helps in identifying the mechanism that applies.

Indeed, we concluded that if the mechanism involves two sequential ET steps followed by just one chemical step to close the cycle $\left(E_{E} C_{r}\right)$, the two catalytic potentials are equal to the values of the redox potentials determined in the absence of catalysis: $E_{\text {cat }}^{n=1, \text { ox }}=E_{1}^{0}$ and $E_{\text {cat }}^{n=1, \text { red }}=E_{2}^{0}$ (there is no kinetic shift between the catalytic potentials and the non-catalytic potentials). If the mechanism involves two sequential ET steps followed by two or more chemical steps to close the cycle $\left(E E C_{r} C_{r}, E E C_{r} C_{r} C_{r}\right)$, each of the two catalytic potentials is kinetically shifted inward, in the direction of the other from its value in the absence of catalysis, $E_{\text {cat }}^{n=1, \text { ox }}<E_{1}^{0}$ and $E_{\text {cat }}^{n=1 \text {,red }}>E_{2}^{0}$, as shown in figure $3 C$. The reason for each catalytic potential to be shifted in the direction of lower driving force is the same as in the $E C_{i} C_{i}$ mechanism when the 1 st $C_{i}$ step is faster than the second and pulls the $E$ step $^{31,36}$. By contrast, the $\mathrm{EC}_{\mathrm{r}} \mathrm{EC}_{\mathrm{r}}$ mechanism may be easily identified from the observation that either only one redox process is seen in the absence of catalysis, or, if two redox processes are observed, the two catalytic waves are not in between the two non-catalytic potentials.

\section{The kinetic determinants of reversibility}

Another way of using the results in Table 1 is to deduce which properties of the catalysts and of the catalytic cycle (redox potentials and rate constants) define these two catalytic potentials, and how they should be tuned to achieve reversible bidirectional catalysis.

Abundant catalysis literature suggests that "efficient catalysis" requires that all catalytic intermediates have a similar free energy and low barriers, so that the energy landscape is flat rather than characterized by deep sinks and high energy intermediates; indeed, large 
free energy differences along the reaction coordinates should imply high energy barriers ${ }^{56}$ and thus kinetic penalties. This idea is largely supported by the examination of volcano plots in heterogeneous catalysis, which demonstrate that the binding of the primary catalytic intermediate on the surface should be neither too strong nor too weak. According to this design principle, each chemical step in the catalytic cycle should have an equilibrium constant $\left(k_{i} / k_{-i}\right)$ close to one; this design principle is explicit in discussions of heterogeneous (materials) catalysis, according to which each reaction step must be thermoneutral to maximize the exchange current density. ${ }^{57,58}$

In molecular electrocatalysis, it is generally accepted that smoothing the free energy landscape should improve the catalyst efficiency. However, designing a molecular catalyst that perfectly adheres to the Sabatier principle is a daunting task, and real catalysts will inevitably possess some high and low energy intermediates along the reaction coordinate. If smoothness is required for unidirectional catalysts, the assumption is that it is even more important for attaining bidirectionality and reversibility. To evaluate if the energy landscape needs to be flat, and which properties contribute to controlling that, we successively discuss bidirectionality, rate and reversibility, and we show in particular that under certain conditions, making the landscape less flat does favor reversibility.

Examination of Table 1 shows that there is no rule according to which every $K_{i}$ must be close to 1 to achieve bidirectionality. According to eq. 6 in Table 1, in the $E_{E} C_{r} C_{r}$ mechanism, a ratio of limiting currents close to unity implies that the combined chemical steps be thermoneutral $\left(K_{1} K_{2}=1\right.$ ) only if $k_{-2}$ and $k_{1}$ are small, that is, only if the intermediate $\mathrm{X}_{2}$ is very unstable. Even in this case, however, obtaining a ratio of limiting currents equal to one does not require that both $K_{1}$ and $K_{2}$ be equal to 1 . In the $\mathrm{EC}_{\mathrm{r}} \mathrm{EC}_{\mathrm{r}}$ mechanism, the catalytic preference is simply the ratio of the slowest rate constants in either direction (it equates the lowest value of $k_{-2}$ and $k_{-1}$, divided by the lowest value of $k_{2}$ and $k_{1}$ ), and it may or may not be equal to $K_{1}$ or $K_{2}$. If the catalytic preference does equal either $K_{1}$ or $K_{2}$, a preference close to one implies that only one chemical step is thermoneutral; if it does not (e.g. if it equals $k_{1} / k_{-2}$ ), then a value of the preference close to one is not related to any step being thermoneutral. 7,8

However, equilibrium constants very different from 1 result in a kinetic penalty in one of the directions of the reaction, and considering that rate constants for chemical steps are slower than $10^{12} \mathrm{~s}^{-1}$ (typical vibrational frequency), observing significant rates (e.g. greater than $1 \mathrm{~s}^{-1}$ ) in both directions implies that every equilibrium constant must be lower than $10^{12}$, that is 16 $\mathrm{kcal} / \mathrm{mol}$. Reasoning on rates (and using the Eyring equation) rather than on equilibrium constants, we also conclude that every energy barrier must also be lower than $16 \mathrm{kcal} / \mathrm{mol}$. Considering intermolecular chemical steps only, diffusion controlled rate constants are slower than $10^{9} \mathrm{~s}^{-1}$ and the limit becomes $12 \mathrm{kcal} / \mathrm{mol}$.

One may reason instead that a flat energy landscape calculated at zero driving force is a requirement for reversibility (as defined here, fast rate in either direction as a result of a minimal overpotential), however, we can demonstrate that this is not the case. Indeed, regarding the $E E C_{r} C_{r}$ mechanism for example, the two catalytic potentials can be brought 
closer together (making the signal more reversible) in two ways, neither of which flattens the energy landscape. First, destabilizing the product of the first electron transfer $\left(A_{1}\right)$ leads to an inversion of the two one electron catalytic potentials. Second, the first chemical product $\left(\mathrm{X}_{2}\right)$ can be stabilized ( $K_{1} \gg 1, K_{2} \ll 1$ ), leading to a thermodynamic well. These simple conclusions are not true for the $\mathrm{EC}_{\mathrm{r}} \mathrm{EC}_{\mathrm{r}}$ mechanism, but other strategies are discussed below.

The usefulness of using volcano plots to optimize molecular catalysis has been questioned, in particular because they rely on an oversimplified definition of the properties of the system by a single descriptor, such as a binding constant or the equilibrium constant for a particular step in the catalytic cycle. ${ }^{59}$ The activity of a unidirectional molecular electrocatalyst is usually described using two parameters: the mid-wave potential of the catalytic wave (relative to the equilibrium potential) and the limiting turnover frequency on the plateau at high overpotential ${ }^{32,60-62}$ Here, we have emphasized that the definition of three parameters, rather than two, is required to fully characterize a bidirectional catalyst -- the limiting turnover frequency in one direction, the catalytic preference (the ratio of the limiting currents or TOFs in the two directions), and the reversibility -- there is no reason why they should all correlate with the value of a single descriptor, except maybe in a series of very similar catalysts. If a flat landscape is not a requirement for observing bidirectionality and reversibility, reasonably small equilibrium constants and energy barriers are required for the turnover frequencies in both directions to be significant. This points to a major difficulty: it will be hard to optimize all the properties of a catalyst at the same time (reversibility, bidirectionality and turnover frequency).

Figure 5 illustrates the above discussion for the EEC $C_{r} C_{r}$ mechanism in the case of a diffusive catalyst, and shows that there is no obvious relation between reversibility and the flatness of the energy profile. We chose three sets of rate constants (SI Table S1) and in each case we calculated the the voltammograms (in the adsorbed and diffusive cases), and the energy landscapes. The reaction pathways go from catalyst $+2 \mathrm{H}^{+}+2 \mathrm{e}-$ (left) to catalyst $+\mathrm{H}_{2}$ (right), starting with two electron transfer steps, ending with two chemical steps. There is no overall energy difference between substrate and product at the equilibrium potential, where the rate of the reaction is zero (panels A3, B3 and C3); the energy landscapes plotted at the bottom are calculated at $E-E_{\mathrm{eq}}=-300$ and $+300 \mathrm{mV}$, to illustrate the effect of forcing the reaction in one particular direction. The energy difference of each step and the height of each barrier were calculated from the values of the rate constants used in the calculation of the voltammograms (see SI Table S1).

The case in blue (middle column) is the situation chosen here as a reference, where catalysis is irreversible. In the $E E C_{r} C_{r}$ mechanism, reversibility can be achieved either by destabilizing the singly reduced active site, " $A_{1}$ " in figure 2 , as illustrated by case $C$ (red) in figure 5 , or by stabilizing the chemical intermediate " $X_{2}$ " in figure 2 , as exemplified in case $A$ (green) in figure 5. Destabilizing the singly reduced state (compare figure 5 panels B3 and $\mathrm{C} 3$ ) is equivalent to crossing the redox potentials $E_{1}^{0}$ and $E_{2}^{0}$. This does not make the energy landscape flatter, but does make the voltammogram more reversible, without affecting the values of the plateau currents. Alternatively, increasing the rate constants $k_{1}$ and $k_{-2}$ (if all other parameters remain the same) stabilizes the intermediate $\mathrm{X}_{2}$, providing a 
kinetic potential shift, and thus also makes the wave more reversible (compare figure 5 panels $B 3$ and $A 3$, noting that here, the difference in limiting current on row 2 results from the shrinking of the diffusion layer, not from a decrease in turnover frequency). Decreasing (rather than increasing) both $k_{1}$ and $k_{-2}$ also makes the wave more reversible, and decreases the currents (not shown).

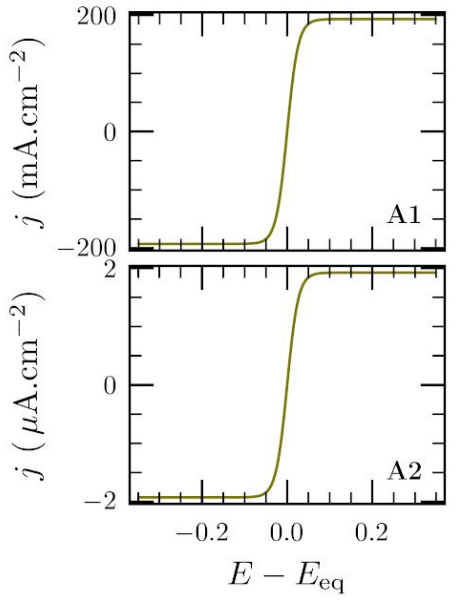

A3

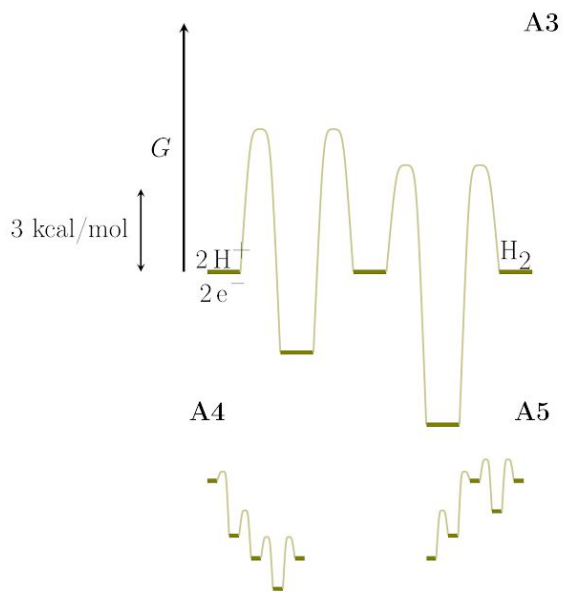

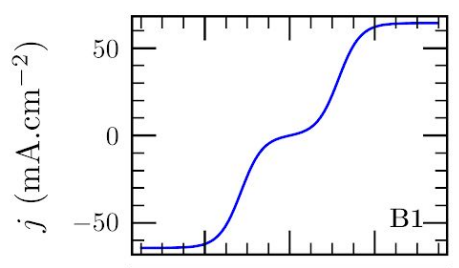
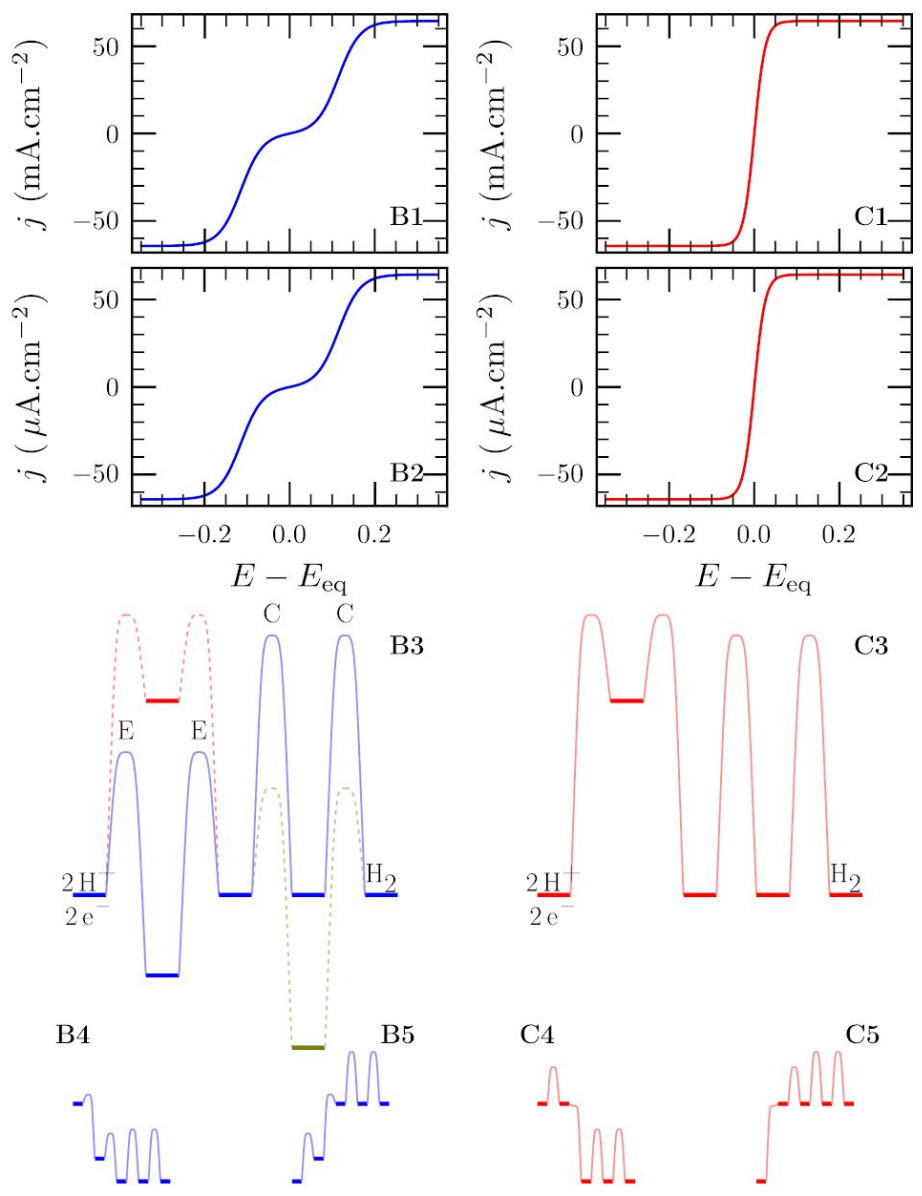

\section{Figure 5}

Relation between voltammetry and energy landscape, in the case of catalysts immobilized (top row, $\Gamma=1 \mathrm{pmol} \cdot \mathrm{cm}^{-2}$ ), and in solution (second row, $D=10^{-6} \mathrm{~cm}^{2} \mathrm{~s}^{-1}$, concentration 1 $\mathrm{mM}$ ) operating by the $\mathrm{EEC}_{\mathrm{r}} \mathrm{C}_{\mathrm{r}}$ mechanism defined in figure 2 . The 3rd row shows the energy landscape at the equilibrium potential (note the ruler on the left). The landscapes shown at the bottom are calculated on the reductive (numbered 4 ) and oxidative (numbered 5) plateau in each of the three situations considered here. Each color corresponds to a set of rate constants, as listed in SI Table S1 (then the energies barriers were simply deduced from the rate constants, using an Eyring equation with transmission coefficient equal to one). Case A (green) was obtained from case B (blue) by increasing the value of the rate constants $k_{1}$ and $k_{-2}$ (keeping all other parameters the same). The change from $B$ (blue) to $C$ (red) illustrates the effect of destabilizing the singly reduced form of the active site (by crossing $E_{1}^{0}$ and $E_{2}^{0}$ ).

A distinct strategy can be employed to improve the performances of catalysts following an $E_{r} E C_{r}$ mechanism (figure 6). For example, starting from a particular situation in which 
$E_{1}^{0}>E_{2}^{0}$ and the chemical steps all have the same rates $\left(k_{1}=k_{-1}=k_{2}=k_{-2}\right)$, it is possible to draw the catalytic potentials closer to one another, or even to cross them, by accelerating the second chemical step (increasing both $k_{2}$ and $k_{-2}$, case $\mathrm{B}$ to case $\mathrm{C}$ in figure 6 ) or slowing down the first chemical step (decreasing $k_{1}$ and $k_{-1}$, case $\mathrm{B}$ to case $\mathrm{A}$ in figure 6). This arises from a "stronger coupling" between the two redox reaction, each pulling the other. Note that slowing the first chemical step ( $B$ to $A$ in figure 6 ) increases the reversibility at the expense of the limiting currents.

Regarding the effect of the equilibrium constants on the reversibility of the wave in the $E_{\mathrm{r}} E C_{\mathrm{r}}$ case, the analysis in SI section S7 shows that if $E_{1}^{0}>E_{2}^{0}$, the value of $K_{2}$ is irrelevant, whereas a value of $K_{1}$ close to one is only required to obtain fully cooperative two-electron wave $\left(E_{\mathrm{cat}}^{n=1, \mathrm{ox}} \ll E_{\mathrm{cat}}^{n=1, \text { red }}\right.$ ). Therefore, making the values of the equilibrium constants closer to 1 has no or little impact on the reversibility of the wave.
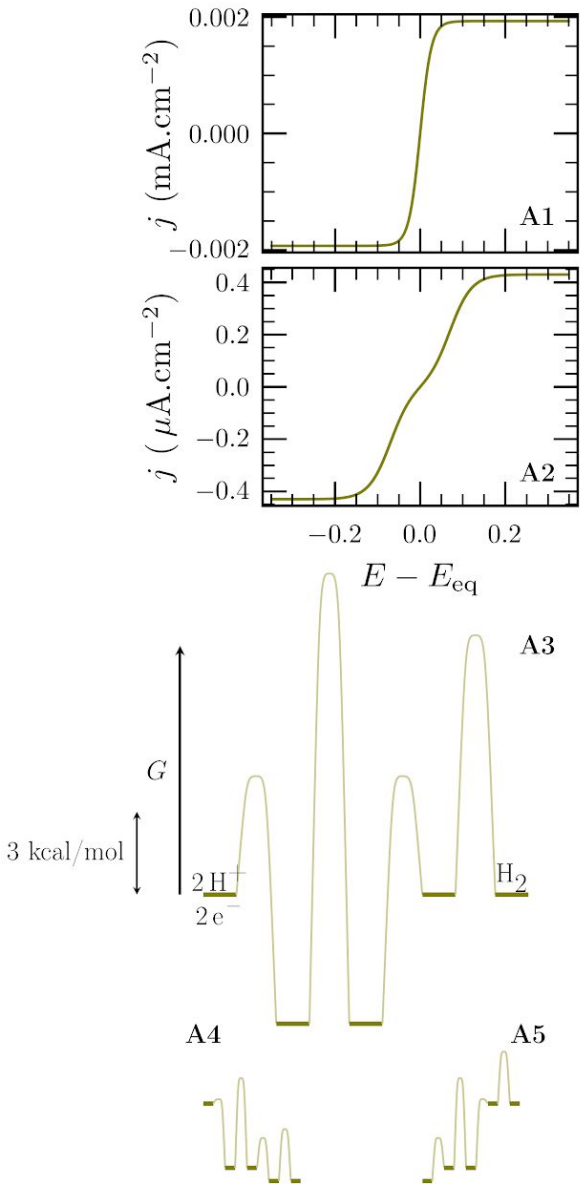

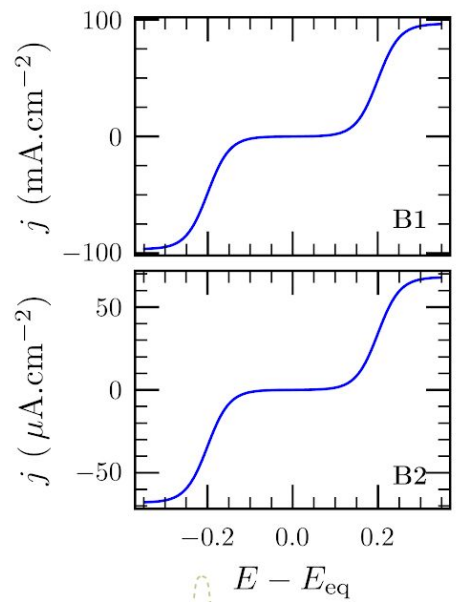

B3

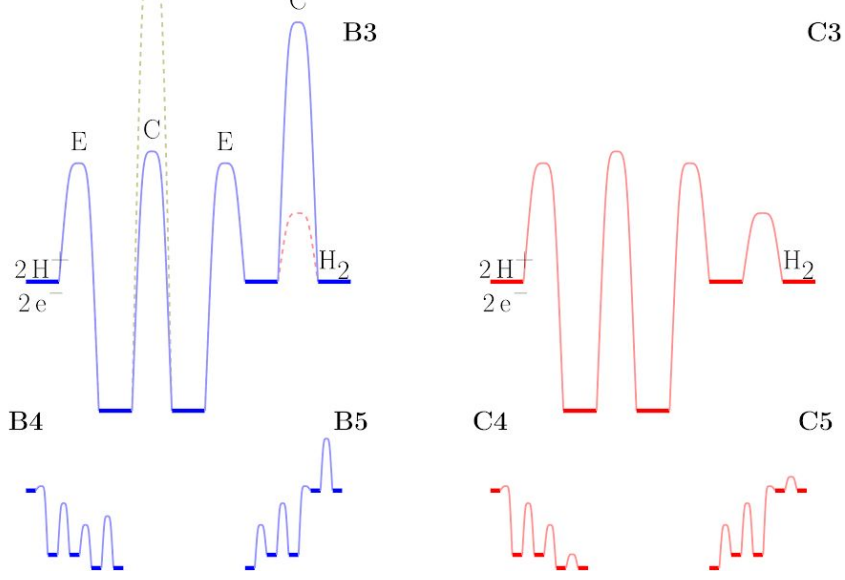

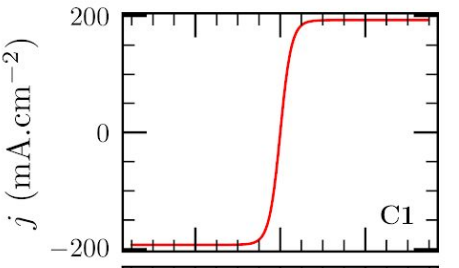

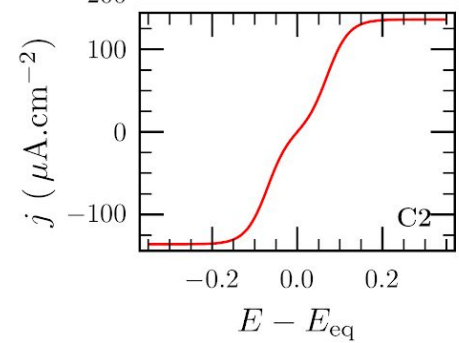

Figure 6.

Relation between voltammetry and energy landscape, in the case of catalysts immobilized (top row, $\Gamma=1 \mathrm{pmol} \cdot \mathrm{cm}^{-2}$ ), and in solution (second row, $D=10^{-6} \mathrm{~cm}^{2} \mathrm{~s}^{-1}$, concentration 1 $\mathrm{mM}$ ) operating by the $\mathrm{EC}_{\mathrm{r}} E \mathrm{C}_{\mathrm{r}}$ mechanism defined in figure 2.

The 3rd row shows the energy landscape at the OCP (note the ruler on the left). The landscapes shown at the bottom are calculated on the reductive (numbered 4) and oxidative (numbered 5) plateau in each of the three situations considered here. Each color

Fourmond et al., 21/33 
corresponds to a set of rate constants, as listed in SI Table S1 (then the energies barriers were simply deduced from the rate constants, using an Eyring equation with transmission coefficient equal to one).

Case A (green) was obtained from case $B$ (blue) by decreasing the values of the rate constants $k_{1}$ and $k_{-1}$ (keeping all other parameters the same). The change from $B$ (blue) to $\mathrm{C}$ (red) illustrates the effect of increasing the rate constants $k_{2}$ and $k_{-2}$.

\section{Illustrations: the bidirectional DuBois catalysts}

DuBois and coworkers have made far-reaching changes in the field of molecular electrocatalysis of $\mathrm{H}_{2}$ by studying a series of soluble mononuclear $\left[\mathrm{Ni}\left(\mathrm{P}_{2} \mathrm{~N}_{2}\right)_{2}\right]^{2+}$ catalysts. ${ }^{63-65}$ These catalysts contain cyclic diphosphine ligands with pendant bases in the second coordination sphere, similar to the structure of the active site of [FeFe]-hydrogenases. ${ }^{4,66}$ Most of the reported $\left[\mathrm{Ni}\left(\mathrm{P}_{2} \mathrm{~N}_{2}\right)_{2}\right]^{2+}$ complexes are unidirectional electrocatalysts for either production or oxidation of $\mathrm{H}_{2}$. Bidirectional and reversible oxidation/production of $\mathrm{H}_{2}$ can be achieved for some catalysts by tuning functional groups on the phosphorus atom in combination with appropriate side chains on the pendant amine. ${ }^{4,67,68}$ These are the only bidirectional molecular electrocatalysts reported to date. Here, we will use the electrocatalytic models developed in this article to evaluate three former examples: 1) a reversible/bidirectional catalyst, 2) the same catalyst as in 1, but under conditions that result in slightly irreversible/bidirectional catalysis, and 3) a very irreversible, bidirectional catalyst.

The first example is the behavior of the CyPhe catalyst in methanol plus $\mathrm{H}_{2} \mathrm{O}$, which shows a reversible/bidirectional catalytic signal (figure 7A) ${ }^{67} \mathrm{CyPhe}$ is a shorthand notation indicating cyclohexyl groups on the phosphorous atoms and phenylalanine groups contributing the nitrogen to the $\mathrm{P}_{2} \mathrm{~N}_{2}$ ligand of a $\left[\mathrm{Ni}\left(\mathrm{P}_{2}^{\mathrm{Cy}}{ }_{2} \mathrm{~N}^{\mathrm{Phe}}{ }_{2}\right)_{2}\right]^{2+}$ catalyst. The derivative of the signal in figure 7B shows that the wave is essentially a single two-electron sigmoid (slightly broadened at high potential), whose two-electron catalytic potential is close to the equilibrium potential, and indeed the catalytic preference is close to unity, consistent with eq. 2. This suggests that the mechanism is ordered. In the absence of $\mathrm{H}_{2}$ and protons, the same catalyst gives the voltammogram in figure 8 , the information from which can be used to identify the operating catalytic mechanism. Controlled potential electrolysis of CyPhe indicates the non catalytic signal results from two overlapping one-electron reactions. As a result, the reversible catalytic signal in figure $7 A$ is best described by an $E C_{r} E C_{r}$ mechanism near the equilibrium potential. Indeed, if catalysis operated according to the EECC mechanism near equilibrium, we would expect a single two-electron wave in between the two one-electron non catalytic potentials, but as seen in figure 7A, the non-catalytic potentials (indicated by a blue dot) are more negative than both catalytic potentials. Therefore, the electrochemical data suggest that around the equilibrium potential, the mechanism is ordered and $\mathrm{EC}_{\mathrm{r}} \mathrm{EC}_{\mathrm{r}}$. Note that there is evidence that in acidic methanol, the catalyst protonates on one of the pendant amines before it enters the two-electron two-proton catalytic cycle ${ }^{69}$ in which case the potential seen in fig 8 corresponds to the redox transition of a precatalytic state, and should not be directly compared with the position of the catalytic wave; however, it is likely that the resulting shift in potential is small enough that the above reasoning holds. 
The second example, the same catalyst but in pure methanol, gives interesting insight and a different conclusion (figure $7 \mathrm{C}$ ). Here a slight irreversibility is evident as a discontinuity in the oxidative wave at $\sim-0.3 \mathrm{~V}$, and two one-electron catalytic potentials are clearly measured from the positions of the peaks in the 1st-derivative of the data (figure 7D). Their average position gives $E_{\text {cat }}^{n=2}$ which is about $50 \mathrm{mV}$ more positive than the equilibrium potential, from which eq 2 predicts a 50 -fold preference in the direction of $\mathrm{H}_{2}$ oxidation that is clearly not observed here. Moreover, eq 1 cannot be fitted to the precise shape of the voltammogram. This means that eqs 1 and 2 do not apply here, suggesting that the catalytic mechanism is branched, rather than ordered, and that the two features in the voltammetry may each correspond to a different pathway.
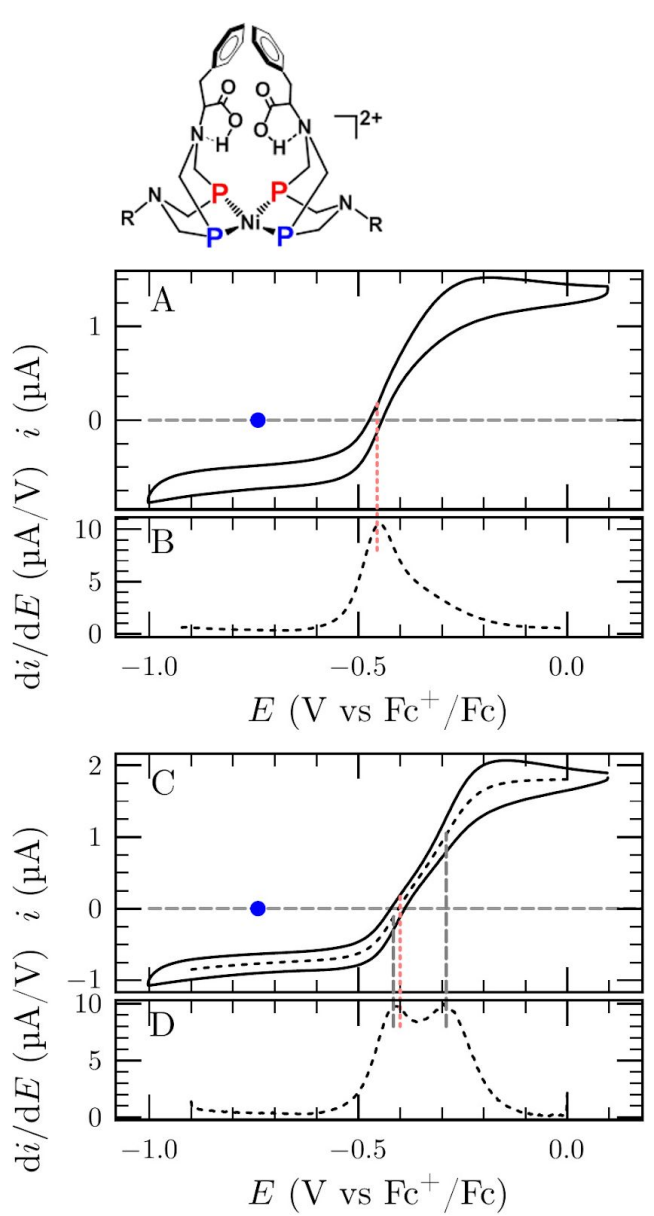

Figure 7

Structure and catalytic voltammetry of the "CyPhe" complex in acidic methanol in the presence $(A, B)$ or absence $(C, D)$ of water. The blue dots mark the position of the non-catalytic peak in methanol only in figure 8. The cyclohexyl groups are not shown for clarity, and the phenylalanine groups are only shown on two ligands for clarity, and as "N-R" on the other two ligands.

Panel A: CV of CyPhe in methanol plus water $\left(\mathrm{C}=0.36 \mathrm{mM}, 25^{\circ} \mathrm{C}, 0.1 \mathrm{M} \mathrm{nBu}_{4} \mathrm{~N}^{+} \mathrm{BF}_{4}^{-}\right.$, and $0.25 \mathrm{~atm} \mathrm{H}_{2}$, with 15 equiv of acid (HTFSI)). The vertical dotted red line indicates the $\mathrm{H}^{+} / \mathrm{H}_{2}$ equilibrium potential; the horizontal line indicates zero current.

Panel $B$ shows the 1st derivative of the CV (averaged from the forward and backward sweeps).

Panel C: CV of CyPhe recorded under the same conditions except that no water was present. The dashed line shows the average of the forward and backward sweeps.

Panel D: 1st derivative of the CV. Adapted from ref ${ }^{67}$. 

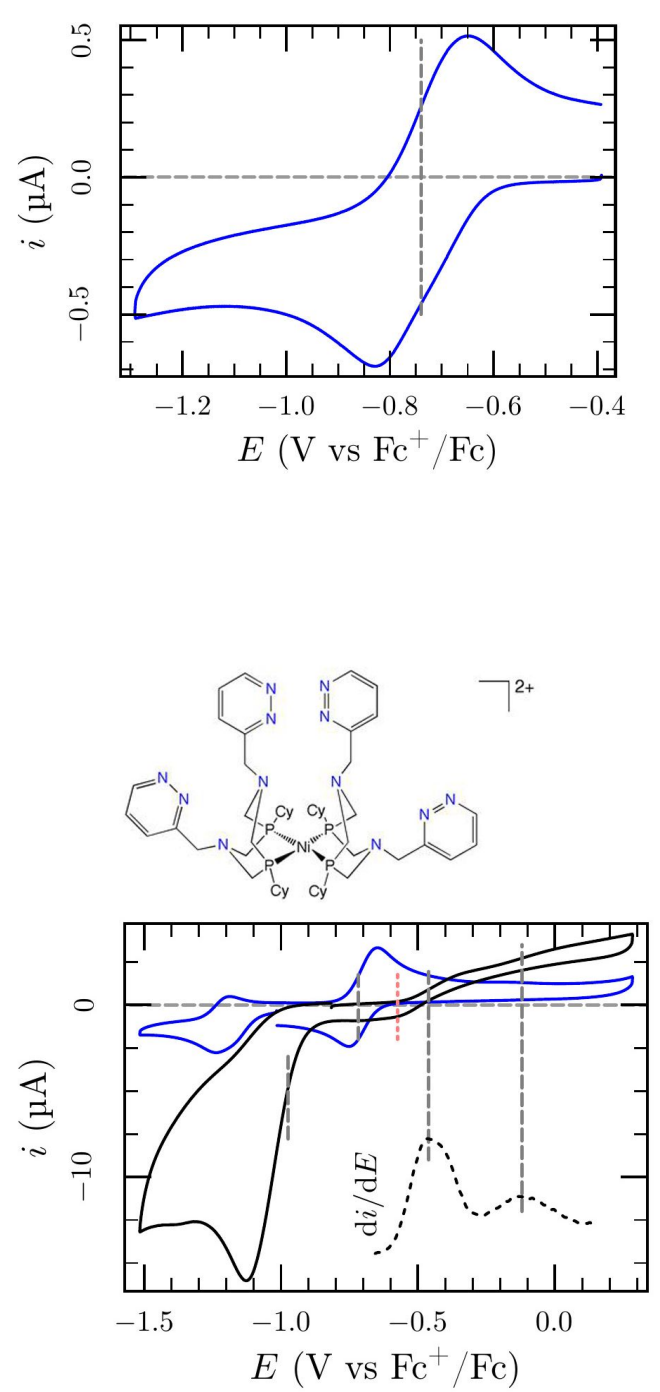

Figure 8. Non-catalytic cyclic voltammetry for CyPhe in methanol on a glassy carbon electrode, adapted from ref ${ }^{67}$.
Figure 9.

Structure and cyclic voltammetry of CyPyz $\left[\mathrm{Ni}\left(\mathrm{P}_{2}^{\mathrm{Cy}} \mathrm{N}_{2 \mathrm{Pz}}\right)_{2}\right]^{2+}$, where Pyz is pyridizine and the cyclohexyl groups are not shown for clarity, in acetonitrile under $\mathrm{N}_{2}$ (blue trace, non-catalytic) and under $\mathrm{H}_{2} / 5.0 \mathrm{M}$ water (black trace, catalytic; with 46 equiv. of $\left[\mathrm{HDMF}^{+}\right)$. The dashed line is the $1 \mathrm{st}$ derivative of the catalytic CV. $\mathrm{C}=0.5 \mathrm{mM}$. Reproduced from ref ${ }^{70}$.

Third, figure 9 shows an example of bidirectional irreversibility for $\mathrm{H}^{+}$reduction and $\mathrm{H}_{2}$ oxidation by a Ni diphosphine catalyst in acetonitrile, called CyPyz, where the Cy refers to cyclohexyl groups on the phosphorous and Pyz refers to pyridizine groups on the nitrogen in a $\left[\mathrm{Ni}\left(\mathrm{P}_{2}^{\mathrm{Cy}}{ }_{2} \mathrm{~N}_{2}^{\mathrm{Pyz}}\right)_{2}\right]^{2+}$ catalyst ${ }^{70}$. From the estimated value of $E_{\text {cat }}^{n=2}$ and the position of the equilibrium potential, eq 2 predicts a $10^{4}$ catalytic preference in the direction of $\mathrm{H}_{2}$ production, clearly not observed here. Again, that eq 2 is defeated suggests that the mechanism is branched. Also we see an additional wave that boosts the current at $-0.1 \mathrm{~V}$ on the oxidative catalytic wave that is not consistent with eq 1 , and reminiscent of signals that are predicted when multiple catalytic pathways are operational (cf our work on molybdo-enzymes ${ }^{37,38,71}$ ). As a side note, the $\mathrm{H}_{2}$ production wave does not attain steady state, indicating additional phenomena (substrate depletion or catalyst decomposition) prevent the maximum rate from being achieved.

Based on the above electrochemical analysis and our previous studies on unidirectional catalysts, ${ }^{45,39,72}$ we propose that the CyPhe catalyst follows the branched mechanism shown in figure 10, where the catalyst follows different pathways depending on the applied potential. We discuss the mechanism under large applied potentials, as well as the mechanism near the equilibrium potential, for both $\mathrm{H}_{2}$ production and $\mathrm{H}_{2}$ oxidation. Sweeping 
the potential causes a gradual transition between the mechanisms that dominate in the specific limiting cases discussed below. Additionally, we will present a hypothesis regarding the role of water in causing the catalytic wave in figure 7 to become more reversible and to appear more consistent with eq 1 (which describes the waveshape for an ordered mechanism).

For production of $\mathrm{H}_{2}$, both ECEC and EECC pathways are possible due to the proximity of the noncatalytic $\mathrm{Ni}(\mathrm{II} / \mathrm{l})$ and $\mathrm{Ni}(\mathrm{I} / 0)$ couples (typically $\Delta E^{\circ}=100-300 \mathrm{mV}$, but is much smaller for CyPhe consistent with figure 8). ${ }^{39,72}$ At electrode potentials sufficiently positive of the $\mathrm{Ni}(I / 0)$ couple, for instance near the equilibrium potential, electron transfer to $\mathrm{Ni}(\mathrm{l})(\mathrm{b} \rightarrow \mathrm{d})$ is prohibitively slow and reductive catalysis operates via the ECEC mechanism $(a \rightarrow b \rightarrow c \rightarrow e)$. At more negative potentials, the $\mathrm{Ni}(\mathrm{l})$ intermediate is reduced by the electrode $(b \rightarrow d)$ faster than it is protonated by the acid $(b \rightarrow c)$, and catalysis proceeds exclusively through the EECC mechanism.

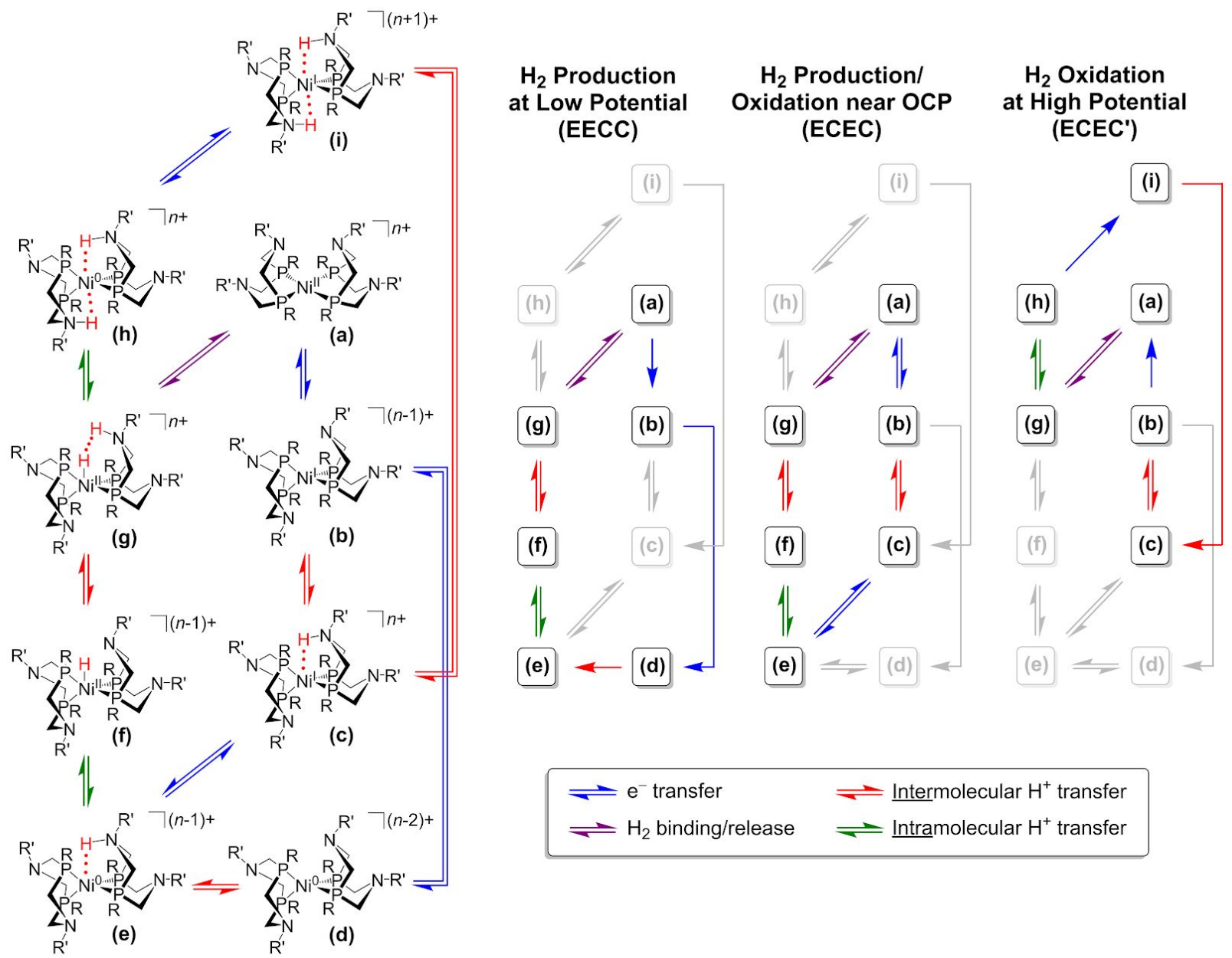

Figure 10

A branched catalytic cycle for the reversible production and oxidation of $\mathrm{H}_{2}$ by the CyPhe catalyst (left). Each of the smaller panels illustrates the dominant pathway for catalysis at different electrode potentials. Each intermediate carries at least one additional proton through the catalytic cycle, likely stored on a carboxylic acid on the pendant amine. ${ }^{69}$ 
For $\mathrm{H}_{2}$ oxidation, CyPhe proceeds through one of two different ECEC mechanisms that are active at different electrode potentials. Near the equilibrium potential, $\mathrm{H}_{2}$ is necessarily oxidized by the same ECEC mechanism that produces $\mathrm{H}_{2}$ at the same potential. ${ }^{45,73}$ In this ECEC pathway, $\mathrm{H}_{2}$ binding $(\mathrm{a} \rightarrow \mathrm{g})$ is followed by removal of the first proton $(\mathrm{g} \rightarrow \mathrm{f})$ prior to oxidation of the catalyst $(\mathrm{f} \rightarrow \mathrm{e} \rightarrow \mathrm{c}$ ). At higher potentials, a second pathway (ECEC') is possible in which intramolecular isomerization of the $\mathrm{H}_{2}$ addition product $(\mathrm{g} \rightarrow \mathrm{h})$ allows the catalyst to be oxidized $(\mathrm{h} \rightarrow \mathrm{i})$ prior to removal of the first proton $(\mathrm{i} \rightarrow \mathrm{c}){ }^{43-45}$

In neat methanol with no water present, the ECEC mechanism occurring near the equilibrium potential affords a much smaller current than observed for the ECEC' mechanism at higher potentials, resulting in two very pronounced catalytic waves. When water is present in the solution, the anodic current near the equilibrium is much larger, and the second mechanism may still be observed as a tail (figure 7B). This suggests that water is facilitating proton transport away from $\mathrm{Ni}$ in at least one of the chemical steps (for example, $\mathrm{g} \rightarrow \mathrm{f}$ ), leading to an improvement in the reversibility of catalysis. As a result, in the presence of water, the $\mathrm{CV}$ of CyPhe adheres to equation 1 and the catalyst behaves as if the mechanism followed an ordered mechanism, because the two branches are fast.

A key difference between the two catalysts discussed here is the outer coordination sphere group that facilitates proton transport. For the irreversible/bidirectional catalyst (figure 9), the proton transport group is a pyridazine, while for CyPhe it is a $\mathrm{COOH}$ group. While both groups enable facile proton transport, the relative rates have not been determined in aqueous solvents, the rate of proton transport has only been evaluated for the doubly-protonated $\mathrm{Ni}(0)$ species $(\mathrm{g} \rightarrow \mathrm{h})$, and it is not clear if that is the only role of the proton transfer groups. For instance, the functional groups may accelerate proton transport by modulating the position of the amine through hydrogen bonding. (Indeed, we showed in the discussion of figure 6 that in the $E_{\mathrm{r}_{\mathrm{r}}} \mathrm{C}_{\mathrm{r}}$ mechanism, accelerating one of the two chemical steps without changing any equilibrium constant may increase reversibility.) The steric accessibility and flexibility of the $\mathrm{COOH}$ group may provide structural advantages over the pyridazyl group, which is expected to introduce ring strain upon forming a hydrogen bond with a proton on the pendant amine.

\section{Illustrations: hydrogenases}

The natural catalysts of hydrogen oxidation and production are large (> $60 \mathrm{kDa})$ proteins, that embed a dinuclear, inorganic (NiFe or FeFe) active site which is connected to the solvent by gas channels that guide the diffusion of $\mathrm{H}_{2}$, chains of redox centers (FeS clusters) that mediate long range electron transfers, and acidic amino acids and water molecules that transfer protons. They are easily wired to electrodes, which has allowed many investigations of their mechanistic properties. ${ }^{55,74} \mathrm{~A}$ straightforward observation in protein electrochemistry experiments is that all hydrogenases catalyse both $\mathrm{H}_{2}$ production and oxidation, although the values of the catalytic preference differ, and some NiFe hydrogenases reduce protons only at low $\mathrm{pH}$. Their mechanism involves sites of the protein that are remote from the active site, and the catalytic cycle could barely be reduced to a 4-step mechanism such as those in 
figure 2. And yet various observations previously made with hydrogenases and hydrogenase mutants illustrate some aspects of the above discussion.

First, with the enzymes immobilized onto a rotating disc electrode, all catalytic waveshapes are described well by eq 1 . (see e.g. figure 7 in ref ${ }^{33}$, and 3 in ref ${ }^{8}$ ), on the condition that experimental conditions are chosen that prevent hydrogen depletion near the electrode, low ${ }^{75}$ or high potential ${ }^{76,77,78}$ inactivation of the enzyme, and provided that the complications that arise from slow interfacial electron transfer are taken into account. ${ }^{33,48}$ Intramolecular electron transfer also affects the wave shape on condition that interfacial electron transfer is not infinitely fast, so that very accurate fitting procedures can evidence the presence of functional intramolecular electron transfer relays. ${ }^{8,33}$ A series of mutations have been identified that tune the catalytic bias without affecting the redox properties of the active site (figure 11), demonstrating that the catalytic preference of $D$. fructosovorans [NiFe] hydrogenase does not depend only on the active site's redox potential. ${ }^{7}$ Similarly, the observation by Parkin and co-workers that modifying the distal cluster of $E$. coli NiFe hydrogenase biases the enzyme in the direction of $\mathrm{H}_{2}$ production suggests that the modification slows intramolecular electron transfer, which is rate limiting in the direction of $\mathrm{H}_{2}$ oxidation. ${ }^{11}$. But there are also recent examples in the litterature, mainly regarding mutagenesis studies of FeFe hydrogenases by Rüdiger and co-workers, according to which modifications of the immediate environment of the active site also drastically affects the



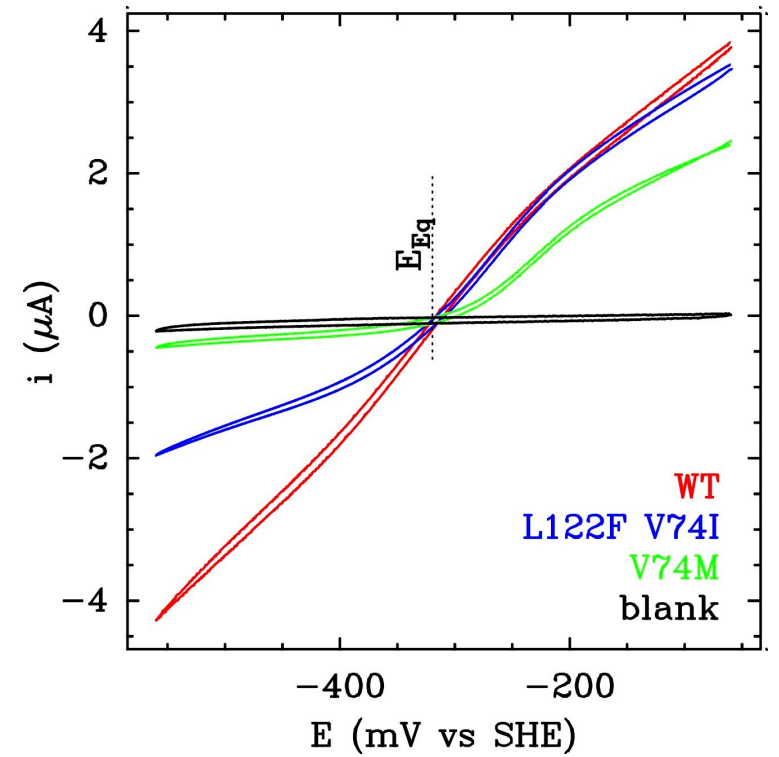

Figure 11

Bidirectional electrocatalysis by the WT (red), L122F-V74I (blue), and V74 M (green) forms of $D$. fructosovorans [NiFe] hydrogenase. At the equilibrium potential, the rates of oxidation and reduction exactly cancel each other and the current is zero. The blank was recorded with no adsorbed enzyme. Reprinted from ref ${ }^{7}$, with permission.

A well known characteristic of many [NiFe]-hydrogenases (including those well studied from $D$. gigas and $D$. fructosovorans) is that the chain of FeS clusters that wires the active site to the redox partner of the enzyme consists of a low potential [4Fe4S] cluster that is proximal to the active site, a medial high potential [3Fe4S] cluster, and a distal low potential [4Fe4S] cluster. The potential difference is as large as $400 \mathrm{mV}$, which creates a $9 \mathrm{kcal} / \mathrm{mol}$ - deep sink in the electron transfer process. The involvement of the high potential cluster in electron transfer was actually questioned when the structure of the enzyme was first solved, ${ }^{79}$ this sink challenging the idea that enzymes act by avoiding high energy intermediates. And yet 
this high-potential cluster is actually in the middle of the electron transfer chain, which does not prevent [NiFe]-hydrogenases from being bidirectional, reversible and fast catalysts. This is probably the most famous and spectacular demonstration that flat energy landscapes are not required for fast and efficient catalysis. The solution to the paradox is now well known: that some steps in the catalytic cycle are very endergonic is not an issue provided they are fast and combined with exergonic steps that drive the overall reaction. ${ }^{80}$

In discussing Table 1, we have emphasized that irrespective of which mechanism applies, the catalytic bias may be the equilibrium constant of one of the chemical steps or the product of the equilibrium constants of the two chemical steps, but it may also be defined by the ratio of two rate constants that do not relate to the same step. Of course in the general case it is a combination of all rate constants in the model. Recent investigations of ours identified two cases where the former limiting cases apply. In [NiFe]-hydrogenases, regarding solution assays with methyl viologen as an electron partner, there is a consensus that intermolecular electron transfer is the rate limiting step only in the direction of $\mathrm{H}_{2}$ oxidation. Hence modifying the electron transfer chain slows $\mathrm{H}_{2}$ oxidation without affecting $\mathrm{H}_{2}$ production. ${ }^{81}$ In contrast, intramolecular $\mathrm{H}_{2}$ diffusion out of the enzyme is the rate limiting step of $\mathrm{H}_{2}$ production in some site-directed mutants, and blocking the gas channel tunes the catalytic bias in the direction of $\mathrm{H}_{2}$ oxidation. ${ }^{7,81}$ These two examples illustrate the case where the catalytic bias is not the equilibrium constant of one particular step in the catalytic cycle. However, the latter situation seems to occur in Megasphaera elsdenii FeFe hydrogenase, where the analysis of the voltammetry suggests that one particular intramolecular electron transfer steps is rate limiting in both directions of the reaction, and the catalytic bias is the equilibrium constant of that particular ET step, which must be reasonably thermoneutral for the enzyme to function in both directions at a significant rate. ${ }^{8}$

\section{Conclusion}

Here we have fully described the electrochemical signatures of bidirectional catalysts of two electron reactions, that are either adsorbed onto an electrode or diffusing in solution. We emphasized the differences between bidirectional and reversible, ordered and branched mechanisms. We used the theoretical prediction to discuss which properties of the catalytic cycle make the catalytic response bidirectional and reversible, and we gave various diagnostic criteria that can be easily applied to real data. We have also emphasized the limits of various, common assumptions, for example that interfacial electron transfer is fast and the mechanism is ordered; these will have to be assessed on a case by case basis.

However, there is still much remaining to be done. At the present time, the complexity of competing pathways and additional chemical steps is difficult to capture in kinetic models ${ }^{37,82}$ and to control in synthetic catalysts. Further, the development of additional molecular catalysts that operate bidirectionally can help us to challenge the mechanistic assumptions we have relied upon here. Of additional interest is a more philosophical question about the "best" way to design reversible synthetic catalysts. Most typically, this is approached from the viewpoint of lowering the overpotential of a unidirectional catalyst in order to bring it closer to the equilibrium potential. This approach is usually accompanied by a concomitant decrease in rate, though in some cases this can be circumvented through control of the 
second and outer coordination spheres. ${ }^{4,69,83-87}$ However, a different approach is to begin with a catalyst that displays a bidirectional/irreversible response, and modify the catalyst in a way that brings the two catalytic potentials closer together. It is unclear yet which is the best strategy, due in part to the lack of bidirectional synthetic catalysts, but provides an alternative approach to catalyst design that could allow for unique possibilities.

The present work emphasizes that a highly branched mechanism can behave in voltammetric experiments as a simple ordered mechanism (eq. 1). Because of our existing mechanistic knowledge of unidirectional $\left[\mathrm{Ni}\left(\mathrm{P}_{2} \mathrm{~N}_{2}\right)_{2}\right]^{2+}$ catalysts, we were able to consider how the CyPhe catalyst transitions between multiple pathways as the electrode potential is varied. Instead, if we had no prior knowledge of the mechanism, then analysis of the reversible catalysis by CyPhe would have led us to conclude that a single pathway was operative over the entire potential range of catalysis. This observation has implications for hydrogenase and other reversible enzymes - even though they can frequently be described by relatively simple two-electron models, in reality they may possess many parallel pathways or transition between pathways as a function of the electrode potential.

\section{Associated content}

\section{Author Information}

Corresponding authors: * vincent.fourmond@imm.cnrs.fr, christophe.leger@imm.cnrs.fr

\section{Author contributions}

The manuscript was written through contributions of all authors.

\section{Supporting Information}

The Supporting Information is available free of charge on the ACS Publications website: analytical method for computing the currents, demonstration that all ordered mechanisms give eq. 1 , demonstrations of the results in Table 1 (regarding the case of a catalyst diffusing in solution for the $E E C_{r}, E E C_{r} C_{r}$ and $E C_{r} E C_{r}$ mechanisms), and numerical investigation of the $E E C_{r} C_{r} C_{r}$ and $E C_{r} E C_{r}$ mechanisms.

\section{Acknowledgements}

The French team is supported by CNRS, Aix Marseille Université, Nationale de la Recherche (ANR-12-BS08-0014, ANR-14-CE05-0010, ANR-17-CE11-0027) and the Excellence Initiative of Aix-Marseille University - A*MIDEX, a French "Investissements d'Avenir" programme (ANR-11-IDEX-0001-02). The French authors are part of the French bioinorganic chemistry network. E.S.W. was supported by the Center for Molecular Electrocatalysis, an Energy Frontier Research Center funded by the U.S. Department of Energy (USDOE), Office of Science, Office of Basic Energy Sciences (BES). The work discussed here was originally supported for WJS by the Office of Science Early Career Research Program through the USDOE, with current funding from the USDOE, BES, Chemical Sciences, Geosciences, and Biosciences program.

\section{References}

(1) Rountree, E. S.; McCarthy, B. D.; Eisenhart, T. T.; Dempsey, J. L. Evaluation of Homogeneous Electrocatalysts by Cyclic Voltammetry. Inorg. Chem. 2014, 53 (19), 9983-10002. doi: 10.1021/ic500658x.

(2) Lee, K. J.; Elgrishi, N.; Kandemir, B.; Dempsey, J. L. Electrochemical and Spectroscopic Methods for 
Evaluating Molecular Electrocatalysts. Nature Reviews Chemistry 2017, 1 (5), 0039. doi: 10.1038/s41570-017-0039.

(3) Costentin, C.; Savéant, J.-M. Homogeneous Molecular Catalysis of Electrochemical Reactions. Scaling Relations, Intrinsic and Operational Factors. J. Am. Chem. Soc. 2018. doi: 10.1021/jacs.8b09154.

(4) Dutta, A.; Appel, A. M.; Shaw, W. J. Designing Electrochemically Reversible $\mathrm{H}_{2}$ Oxidation and Production Catalysts. Nat Rev Chem 2018, 2 (9), 244-252. doi: 10.1038/s41570-018-0032-8.

(5) Armstrong, F. A.; Hirst, J. Reversibility and Efficiency in Electrocatalytic Energy Conversion and Lessons from Enzymes. Proc. Natl. Acad. Sci. U. S. A. 2011, 108 (34), 14049-14054. doi: 10.1073/pnas.1103697108.

(6) Léger, C.; Bertrand, P. Direct Electrochemistry of Redox Enzymes as a Tool for Mechanistic Studies. Chem. Rev. 2008, 108 (7), 2379-2438. doi: 10.1021/cr0680742.

(7) Abou Hamdan, A.; Dementin, S.; Liebgott, P.-P.; Gutierrez-Sanz, O.; Richaud, P.; De Lacey, A. L.; Rousset, M.; Bertrand, P.; Cournac, L.; Léger, C. Understanding and Tuning the Catalytic Bias of Hydrogenase. J. Am. Chem. Soc. 2012, 134 (20), 8368-8371. doi: 10.1021/ja301802r.

(8) Caserta, G.; Papini, C.; Adamska-Venkatesh, A.; Pecqueur, L.; Sommer, C.; Reijerse, E.; Lubitz, W.; Gauquelin, C.; Meynial-Salles, I.; Pramanik, D.; Artero, V.; Atta, M.; del Barrio, M.; Faivre; B.; Fourmond, V.; Léger, C.; Fontecave, M. Engineering an [FeFe]-Hydrogenase: Do Accessory Clusters Influence $\mathrm{O} 2$ Resistance and Catalytic Bias? J. Am. Chem. Soc. 2018, 140 (16), 5516-5526. doi: 10.1021/jacs.8b01689.

(9) McIntosh, C. L.; Germer, F.; Schulz, R.; Appel, J.; Jones, A. K. The [NiFe]-Hydrogenase of the Cyanobacterium Synechocystis Sp. PCC 6803 Works Bidirectionally with a Bias to H2 Production. J. Am. Chem. Soc. 2011, 133 (29), 11308-11319. doi: 10.1021/ja203376y.

(10) Lampret, O.; Adamska-Venkatesh, A.; Konegger, H.; Wittkamp, F.; Apfel, U.-P.; Reijerse, E. J.; Lubitz, W.; Rüdiger, O.; Happe, T.; Winkler, M. Interplay between CN Ligands and the Secondary Coordination Sphere of the H-Cluster in [FeFe]-Hydrogenases. J. Am. Chem. Soc. 2017, 139 (50), 18222-18230. doi: $10.1021 /$ jacs.7b08735.

(11) Adamson, H.; Robinson, M.; Wright, J. J.; Flanagan, L. A.; Walton, J.; Elton, D.; Gavaghan, D. J.; Bond, A. M.; Roessler, M. M.; Parkin, A. Retuning the Catalytic Bias and Overpotential of a [NiFe]-Hydrogenase via a Single Amino Acid Exchange at the Electron Entry/Exit Site. J. Am. Chem. Soc. 2017, 139 (31), 10677-10686. doi: 10.1021/jacs.7b03611.

(12) Rodríguez-Maciá, P.; Kertess, L.; Burnik, J.; Birrell, J. A.; Hofmann, E.; Lubitz, W.; Happe, T.; Rüdiger, O. His-Ligation to the [4Fe-4S] Sub-Cluster Tunes the Catalytic Bias of [FeFe] Hydrogenase. J. Am. Chem. Soc. 2019, 141 (1), 472-481. doi: 10.1021/jacs.8b11149.

(13) Therien, J. B.; Artz, J. H.; Poudel, S.; Hamilton, T. L.; Liu, Z.; Noone, S. M.; Adams, M. W. W.; King, P. W.; Bryant, D. A.; Boyd, E. S.; Peters, J. W. The Physiological Functions and Structural Determinants of Catalytic Bias in the [FeFe]-Hydrogenases Cpl and Cpll of Strain W5. Front. Microbiol. 2017, 8, 1305. doi: 10.3389/fmicb.2017.01305.

(14) Murphy, B. J.; Sargent, F.; Armstrong, F. A. Transforming an Oxygen-Tolerant [NiFe] Uptake Hydrogenase into a Proficient, Reversible Hydrogen Producer. Energy Environ. Sci. 2014, 7 (4), 1426-1433. doi: 10.1039/C3EE43652G.

(15) Kertess, L.; Adamska-Venkatesh, A.; Rodríguez-Maciá, P.; Rüdiger, O.; Lubitz, W.; Happe, T. Influence of the [4Fe-4S] Cluster Coordinating Cysteines on Active Site Maturation and Catalytic Properties of [FeFe]-Hydrogenase. Chem. Sci. 2017, 8 (12), 8127-8137. doi: 10.1039/c7sc03444j.

(16) We call "overpotential", the difference between the electrode potential and the Nernst potential of the redox couple whose transformation is catalyzed, so that the net reaction rate Is zero when the overpotential is zero.

(17) Pershad, H. R.; Duff, J. L.; Heering, H. A.; Duin, E. C.; Albracht, S. P.; Armstrong, F. A. Catalytic Electron Transport in Chromatium Vinosum [NiFe]-Hydrogenase: Application of Voltammetry in Detecting Redox-Active Centers and Establishing That Hydrogen Oxidation Is Very Fast Even at Potentials close to the Reversible $\mathrm{H}^{+} / \mathrm{H}_{2}$ Value. Biochemistry 1999, 38 (28), 8992-8999. doi: 10.1021/bi990108v.

(18) Léger, C.; Heffron, K.; Pershad, H. R.; Maklashina, E.; Luna-Chavez, C.; Cecchini, G.; Ackrell, B. A. C.; Armstrong, F. A. Enzyme Electrokinetics: Energetics of Succinate Oxidation by Fumarate Reductase and Succinate Dehydrogenase †. Biochemistry 2001, 40 (37), 11234-11245. doi: 10.1021/bi010889b.

(19) Wang, V. C.-C.; Can, M.; Pierce, E.; Ragsdale, S. W.; Armstrong, F. A. A Unified Electrocatalytic Description of the Action of Inhibitors of Nickel Carbon Monoxide Dehydrogenase. J. Am. Chem. Soc. 2013, 135 (6), 2198-2206. doi: 10.1021/ja308493k.

(20) Bassegoda, A.; Madden, C.; Wakerley, D. W.; Reisner, E.; Hirst, J. Reversible Interconversion of $\mathrm{CO}_{2}$ and Formate by a Molybdenum-Containing Formate Dehydrogenase. J. Am. Chem. Soc. 2014, 136 (44), 15473-15476. doi: 10.1021/ja508647u.

(21) Zu, Y.; Shannon, R. J.; Hirst, J. Reversible, Electrochemical Interconversion of NADH and NAD+ by the Catalytic (lambda) Subcomplex of Mitochondrial NADH:ubiquinone Oxidoreductase (complex I). J. Am. Chem. Soc. 2003, 125 (20), 6020-6021. doi: 10.1021/ja0343961.

(22) Kurth, J. M.; Dahl, C.; Butt, J. N. Catalytic Protein Film Electrochemistry Provides a Direct Measure of the Tetrathionate/Thiosulfate Reduction Potential. J. Am. Chem. Soc. 2015, 137 (41), 13232-13235. doi: 10.1021/jacs.5b08291.

(23) Fersht, A.; University Alan Fersht. Structure and Mechanism in Protein Science: A Guide to Enzyme Catalysis and Protein Folding; W. H. Freeman, 1999. 
(24) Cornish-Bowden, A. Fundamentals of Enzyme Kinetics, 4th Edition; Wiley-Blackwell, 2012.

(25) Maurin, A.; Robert, M. Noncovalent Immobilization of a Molecular Iron-Based Electrocatalyst on Carbon Electrodes for Selective, Efficient $\mathrm{CO}_{2}$-to-CO Conversion in Water. J. Am. Chem. Soc. 2016, 138 (8), 2492-2495. doi: 10.1021/jacs.5b12652.

(26) Rodríguez-Maciá, P.; Priyadarshani, N.; Dutta, A.; Weidenthaler, C.; Lubitz, W.; Shaw, W. J.; Rüdiger, O. Covalent Attachment of the Water-Insoluble Ni(PCy $\left.\mathrm{NPhe}_{2}\right)_{2}$ Electrocatalyst to Electrodes Showing Reversible Catalysis in Aqueous Solution. Electroanalysis 2016, 28 (10), 2452-2458. doi: 10.1002/elan.201600306.

(27) Gentil, S.; Lalaoui, N.; Dutta, A.; Nedellec, Y.; Cosnier, S.; Shaw, W. J.; Artero, V.; Le Goff, A. Carbon-Nanotube-Supported Bio-Inspired Nickel Catalyst and Its Integration in Hybrid Hydrogen/Air Fuel Cells. Angew. Chem. Int. Ed Engl. 2017, 56 (7), 1845-1849. doi: 10.1002/anie.201611532.

(28) Rodriguez-Maciá, P.; Dutta, A.; Lubitz, W.; Shaw, W. J.; Rüdiger, O. Direct Comparison of the Performance of a Bio-Inspired Synthetic Nickel Catalyst and a [NiFe]-Hydrogenase, Both Covalently Attached to Electrodes. Angew. Chem. Int. Ed Engl. 2015, 54 (42), 12303-12307. doi: 10.1002/anie.201502364.

(29) Savéant, J.-M. Molecular Catalysis of Electrochemical Reactions. Cyclic Voltammetry of Systems Approaching Reversibility. ACS Catal. 2018, 8 (8), 7608-7611. doi: 10.1021/acscatal.8b02007.

(30) Hexter, S. V.; Grey, F.; Happe, T.; Climent, V.; Armstrong, F. A. Electrocatalytic Mechanism of Reversible Hydrogen Cycling by Enzymes and Distinctions between the Major Classes of Hydrogenases. Proc. Natl. Acad. Sci. U. S. A. 2012, 109 (29), 11516-11521. doi: 10.1073/pnas.1204770109.

(31) Costentin, C.; Savéant, J.-M. Multielectron, Multistep Molecular Catalysis of Electrochemical Reactions: Benchmarking of Homogeneous Catalysts. ChemElectroChem 2014, 1 (7), 1226-1236. doi: 10.1002/celc.201300263.

(32) Artero, V.; Saveant, J.-M. Toward the Rational Benchmarking of Homogeneous $\mathrm{H}_{2}$-Evolving Catalysts. Energy Environ. Sci. 2014, 7 (11), 3808-3814. doi: 10.1039/C4EE01709A.

(33) Fourmond, V.; Baffert, C.; Sybirna, K.; Lautier, T.; Abou Hamdan, A.; Dementin, S.; Soucaille, P.; Meynial-Salles, I.; Bottin, H.; Léger, C. Steady-State Catalytic Wave-Shapes for 2-Electron Reversible Electrocatalysts and Enzymes. J. Am. Chem. Soc. 2013, 135 (10), 3926-3938. doi: 10.1021/ja311607s.

(34) Costentin, C.; Drouet, S.; Robert, M.; Savéant, J.-M. Turnover Numbers, Turnover Frequencies, and Overpotential in Molecular Catalysis of Electrochemical Reactions. Cyclic Voltammetry and Preparative-Scale Electrolysis. J. Am. Chem. Soc. 2012, 134 (27), 11235-11242. doi: 10.1021/ja303560c.

(35) Wasylenko, D. J.; Rodríguez, C.; Pegis, M. L.; Mayer, J. M. Direct Comparison of Electrochemical and Spectrochemical Kinetics for Catalytic Oxygen Reduction. J. Am. Chem. Soc. 2014, 136 (36), 12544-12547. doi: 10.1021/ja505667t.

(36) For unidirectional catalysis and on condition that all chemical steps are irreversible, analyzing the foot of the wave by mistakenly assuming that the catalytic potential is the potential of the catalyst returns the rate of the chemical step that immediately follows the redox step, instead of the rate of the rate limiting step (cf e. g. the discussion of Fig 6 in ref 1).

(37) Bertrand, P.; Frangioni, B.; Dementin, S.; Sabaty, M.; Arnoux, P.; Guigliarelli, B.; Pignol, D.; Léger, C. Effects of Slow Substrate Binding and Release in Redox Enzymes: Theory and Application to Periplasmic Nitrate Reductase. J. Phys. Chem. B 2007, 111 (34), 10300-10311. doi: 10.1021/jp074340j.

(38) Frangioni, B.; Arnoux, P.; Sabaty, M.; Pignol, D.; Bertrand, P.; Guigliarelli, B.; Léger, C. In Rhodobacter Sphaeroides Respiratory Nitrate Reductase, the Kinetics of Substrate Binding Favors Intramolecular Electron Transfer. J. Am. Chem. Soc. 2004, 126 (5), 1328-1329. doi: 10.1021/ja0384072.

(39) Ho, M.-H.; Rousseau, R.; Roberts, J. A. S.; Wiedner, E. S.; Dupuis, M.; DuBois, D. L.; Bullock, R. M.; Raugei, S. Ab Initio-Based Kinetic Modeling for the Design of Molecular Catalysts: The Case of $\mathrm{H}_{2}$ Production Electrocatalysts. ACS Catal. 2015, 5 (9), 5436-5452. doi: 10.1021/acscatal.5b01152.

(40) Rountree, E. S.; Dempsey, J. L. Potential-Dependent Electrocatalytic Pathways: Controlling Reactivity with pKa for Mechanistic Investigation of a Nickel-Based Hydrogen Evolution Catalyst. J. Am. Chem. Soc. 2015, 137 (41), 13371-13380. doi: 10.1021/jacs.5b08297.

(41) Appel, A. M.; Pool, D. H.; O'Hagan, M.; Shaw, W. J.; Yang, J. Y.; Rakowski DuBois, M.; DuBois, D. L.; Bullock, R. M. [Ni( $\left.\left(\mathrm{PPh}_{2} \mathrm{NBn}_{2}\right)_{2}\left(\mathrm{CH}_{3} \mathrm{CN}\right)\right]^{2+}$ as an Electrocatalyst for $\mathrm{H}_{2}$ Production: Dependence on Acid Strength and Isomer Distribution. ACS Catal. 2011, 1 (7), 777-785. doi: 10.1021/cs2000939.

(42) Horvath, S.; Fernandez, L. E.; Appel, A. M.; Hammes-Schiffer, S. pH-Dependent Reduction Potentials and Proton-Coupled Electron Transfer Mechanisms in Hydrogen-Producing Nickel Molecular Electrocatalysts. Inorg. Chem. 2013, 52 (7), 3643-3652. doi: 10.1021/ic302056j.

(43) Lense, S.; Dutta, A.; Roberts, J. A. S.; Shaw, W. J. A Proton Channel Allows a Hydrogen Oxidation Catalyst to Operate at a Moderate Overpotential with Water Acting as a Base. Chem. Commun. 2014, 50 (7), 792-795. doi: 10.1039/c3cc46829a.

(44) Das, P.; Ho, M.-H.; O’Hagan, M.; Shaw, W. J.; Bullock, R. M.; Raugei, S.; Helm, M. L. Controlling Proton Movement: Electrocatalytic Oxidation of Hydrogen by a nickel(II) Complex Containing Proton Relays in the Second and Outer Coordination Spheres. Dalton Trans. 2014, 43 (7), 2744-2754. doi: 10.1039/c3dt53074d

(45) Yang, J. Y.; Smith, S. E.; Liu, T.; Dougherty, W. G.; Hoffert, W. A.; Kassel, W. S.; Rakowski DuBois, M.; DuBois, D. L.; Bullock, R. M. Two Pathways for Electrocatalytic Oxidation of Hydrogen by a Nickel Bis(diphosphine) Complex with Pendant Amines in the Second Coordination Sphere. J. Am. Chem. Soc. 2013, 135 (26), 9700-9712. doi: 10.1021/ja400705a. 
(46) Canaguier, S.; Fourmond, V.; Perotto, C. U.; Fize, J.; Pécaut, J.; Fontecave, M.; Field, M. J.; Artero, V. Catalytic Hydrogen Production by a Ni-Ru Mimic of NiFe Hydrogenases Involves a Proton-Coupled Electron Transfer Step. Chem. Commun. 2013, 49 (44), 5004-5006. doi: 10.1039/c3cc40987b.

(47) Baffert, C.; Artero, V.; Fontecave, M. Cobaloximes as Functional Models for Hydrogenases. 2. Proton Electroreduction Catalyzed by difluoroborylbis(dimethylglyoximato)cobalt(II) Complexes in Organic Media. Inorg. Chem. 2007, 46 (5), 1817-1824. doi: 10.1021/ic061625m.

(48) Fourmond, V.; Léger, C. Modelling the Voltammetry of Adsorbed Enzymes and Molecular Catalysts. Curr. Op. Electrochem. 2017, 1 (1), 110-120. doi: 10.1016/j.coelec.2016.11.002.

(49) Kadish, K. M.; Sweetland, M.; Cheng, J. S. Electron-Transfer Kinetics of Chlorotetrakis(p-chlorophenyl)porphinatomanganese(III) in Dimethyl Sulfoxide-Imidazole Mixtures. Inorg. Chem. 1978, 17 (10), 2795-2797. doi: 10.1021/ic50188a022.

(50) Mu, X. H.; Schultz, F. A. Correlation of Heterogeneous Electron-Transfer Rate with Electron-Transfer Site in Metalloporphyrins. Inorg. Chem. 1990, 29 (16), 2877-2879. doi: 10.1021/ic00341a001.

(51) Christelle Hureau, Elodie Anxolabéhère-Mallart, Martine Nierlich, Florence Gonnet, Eric Rivière, Geneviève Blondin. Synthesis, Structure and Characterisation of New Phenolato-Bridged Manganese Complexes [L2Mn2]2+ - Formation by Ligand Oxidation in $\mathrm{LaH}[\mathrm{LaH}=$ $\mathrm{N}$-(2-hydroxybenzyl)-N,N'-bis(2-pyridylmethyl)ethane-1,2-diamine]. EurJIC 2002, 2002 (10), 2710-2719.

(52) Hu, I.-F.; Karweik, D. H.; Kuwana, T. Activation and Deactivation of Glassy Carbon Electrodes. J. Electroanal. Chem. Interfacial Electrochem. 1985, 188 (1-2), 59-72. doi: 10.1016/S0022-0728(85)80050-4.

(53) Rice, R. J.; Pontikos, N. M.; McCreery, R. L. Quantitative Correlations of Heterogeneous Electron-Transfer Kinetics with Surface Properties of Glassy Carbon Electrodes. J. Am. Chem. Soc. 1990, 112 (12), 4617-4622. doi: 10.1021/ja00168a001.

(54) Léger, C.; Jones, A. K.; Roseboom, W.; Albracht, S. P. J.; Armstrong, F. A. Enzyme Electrokinetics: Hydrogen Evolution and Oxidation by Allochromatium Vinosum [NiFe]-Hydrogenase. Biochemistry 2002 , 41 (52), 15736-15746. doi: 10.1021/bi026586e.

(55) Sensi, M.; del Barrio, M.; Baffert, C.; Fourmond, V.; Léger, C. New Perspectives in Hydrogenase Direct Electrochemistry. Current Opinion in Electrochemistry 2017, 5 (1), 135-145. doi: 10.1016/j.coelec.2017.08.005.

(56) Nørskov, J. K.; Bligaard, T.; Hvolbaek, B.; Abild-Pedersen, F.; Chorkendorff, I.; Christensen, C. H. The Nature of the Active Site in Heterogeneous Metal Catalysis. Chem. Soc. Rev. 2008, 37 (10), 2163-2171. doi: 10.1039/b800260f.

(57) Koper, M. T. M. Theory of Multiple Proton-electron Transfer Reactions and Its Implications for Electrocatalysis. Chem. Sci. 2013, 4 (7), 2710. doi: 10.1039/c3sc50205h.

(58) Koper, M. T. M.; Bouwman, E. Electrochemical Hydrogen Production: Bridging Homogeneous and Heterogeneous Catalysis. Angew. Chem. Int. Ed Engl. 2010, 49 (22), 3723-3725. doi: 10.1002/anie.201000629.

(59) Costentin, C.; Savéant, J.-M. Towards an Intelligent Design of Molecular Electrocatalysts. Nat. rev. chem. 2017, 1 (11), 0087. doi: 10.1038/s41570-017-0087.

(60) Felton, G. A. N.; Glass, R. S.; Lichtenberger, D. L.; Evans, D. H. Iron-Only Hydrogenase Mimics. Thermodynamic Aspects of the Use of Electrochemistry to Evaluate Catalytic Efficiency for Hydrogen Generation. Inorg. Chem. 2006, 45 (23), 9181-9184. doi: 10.1021/ic060984e.

(61) Fourmond, V.; Jacques, P.-A.; Fontecave, M.; Artero, V. H2 Evolution and Molecular Electrocatalysts: Determination of Overpotentials and Effect of Homoconjugation. Inorg. Chem. 2010, 49 (22), 10338-10347. doi: 10.1021/ic101187v.

(62) Appel, A. M.; Helm, M. L. Determining the Overpotential for a Molecular Electrocatalyst. ACS Catal. 2014, 4 (2), 630-633. doi: 10.1021/cs401013v.

(63) Wilson, A. D.; Newell, R. H.; McNevin, M. J.; Muckerman, J. T.; Rakowski DuBois, M.; DuBois, D. L. Hydrogen Oxidation and Production Using Nickel-Based Molecular Catalysts with Positioned Proton Relays. J. Am. Chem. Soc. 2006, 128 (1), 358-366. doi: 10.1021/ja056442y.

(64) DuBois, D. L. Development of Molecular Electrocatalysts for Energy Storage. Inorg. Chem. 2014, 53 (8), 3935-3960. doi: 10.1021/ic4026969.

(65) Shaw, W. J.; Helm, M. L.; DuBois, D. L. A Modular, Energy-Based Approach to the Development of Nickel Containing Molecular Electrocatalysts for Hydrogen Production and Oxidation. Biochim. Biophys. Acta 2013, 1827 (8-9), 1123-1139. doi: 10.1016/j.bbabio.2013.01.003.

(66) Eberhardt, N. A.; Guan, H. Nickel Hydride Complexes. Chem. Rev. 2016, 116 (15), 8373-8426. doi: 10.1021/acs.chemrev.6b00259.

(67) Priyadarshani, N.; Dutta, A.; Ginovska, B.; Buchko, G. W.; O’Hagan, M.; Raugei, S.; Shaw, W. J. Achieving Reversible $\mathrm{H}_{2} / \mathrm{H}^{+}$Interconversion at Room Temperature with Enzyme-Inspired Molecular Complexes: A Mechanistic Study. ACS Catal. 2016, 6 (9), 6037-6049. doi: 10.1021/acscatal.6b01433.

(68) Smith, S. E.; Yang, J. Y.; DuBois, D. L.; Bullock, R. M. Reversible Electrocatalytic Production and Oxidation of Hydrogen at Low Overpotentials by a Functional Hydrogenase Mimic. Angew. Chem. Int. Ed Engl. 2012, 51 (13), 3152-3155. doi: 10.1002/anie.201108461.

(69) Dutta, A.; DuBois, D. L.; Roberts, J. A. S.; Shaw, W. J. Amino Acid Modified Ni Catalyst Exhibits Reversible $\mathrm{H} 2$ Oxidation/production over a Broad pH Range at Elevated Temperatures. Proc. Natl. Acad. Sci. U. S. A. 2014, 111 (46), 16286-16291. doi: 10.1073/pnas.1416381111.

(70) Dutta, A.; Lense, S.; Roberts, J. A. S.; Helm, M. L.; Shaw, W. J. The Role of Solvent and the Outer 
Coordination Sphere on $\mathrm{H}_{2}$ Oxidation Using $\left[\mathrm{Ni}\left(\mathrm{PCy}_{2} \mathrm{NPyz}_{2}\right) 2\right]^{2+}$ : The Role of Solvent and the Outer Coordination Sphere on H 2 Oxidation. Eur. J. Inorg. Chem. 2015, 2015 (31), 5218-5225. doi: 10.1002/ejic.201500732.

(71) Heffron, K.; Léger, C.; Rothery, R. A.; Weiner, J. H.; Armstrong, F. A. Determination of an Optimal Potential Window for Catalysis by E. Coli Dimethyl Sulfoxide Reductase and Hypothesis on the Role of Mo(V) in the Reaction Pathway. Biochemistry 2001, 40 (10), 3117-3126. doi: 10.1021/bi002452u.

(72) Wiedner, E. S.; Brown, H. J. S.; Helm, M. L. Kinetic Analysis of Competitive Electrocatalytic Pathways: New Insights into Hydrogen Production with Nickel Electrocatalysts. J. Am. Chem. Soc. 2016, 138 (2), 604-616. doi: 10.1021/jacs.5b10853.

(73) Stolley, R. M.; Darmon, J. M.; Helm, M. L. Solvent and Electrolyte Effects on Ni(PR $\left.\mathrm{NR}_{2}^{\prime}\right)_{2}-$ Catalyzed Electrochemical Oxidation of Hydrogen. Chem. Commun. 2014, 50 (28), 3681-3684. doi: 10.1039/C4CC00295D.

(74) Del Barrio, M.; Sensi, M.; Orain, C.; Baffert, C.; Dementin, S.; Fourmond, V.; Léger, C. Electrochemical Investigations of Hydrogenases and Other Enzymes That Produce and Use Solar Fuels. Acc. Chem. Res. 2018, 51 (3), 769-777. doi: 10.1021/acs.accounts.7b00622.

(75) Hajj, V.; Baffert, C.; Sybirna, K.; Meynial-Salles, I.; Soucaille, P.; Bottin, H.; Fourmond, V.; Léger, C. FeFe Hydrogenase Reductive Inactivation and Implication for Catalysis. Energy Environ. Sci. 2014, 7 (2), 715-719. doi: 10.1039/C3EE42075B

(76) Del Barrio, M.; Sensi, M.; Fradale, L.; Bruschi, M.; Greco, C.; de Gioia, L.; Bertini, L.; Fourmond, V.; Léger, C. Interaction of the H-Cluster of FeFe Hydrogenase with Halides. J. Am. Chem. Soc. 2018, 140 (16), 5485-5492. doi: 10.1021/jacs.8b01414.

(77) Jones, A. K.; Lamle, S. E.; Pershad, H. R.; Vincent, K. A.; Albracht, S. P. J.; Armstrong, F. A. Enzyme Electrokinetics: Electrochemical Studies of the Anaerobic Interconversions between Active and Inactive States of Allochromatium Vinosum [NiFe]-Hydrogenase. J. Am. Chem. Soc. 2003, 125 (28), 8505-8514. doi: 10.1021/ja035296y.

(78) Abou Hamdan, A.; Liebgott, P.-P.; Fourmond, V.; Gutiérrez-Sanz, O.; De Lacey, A. L.; Infossi, P.; Rousset, M.; Dementin, S.; Léger, C. Relation between Anaerobic Inactivation and Oxygen Tolerance in a Large Series of NiFe Hydrogenase Mutants. Proc. Natl. Acad. Sci. U. S. A. 2012, 109 (49), 19916-19921. doi: 10.1073/pnas.1212258109.

(79) Volbeda, A.; Charon, M. H.; Piras, C.; Hatchikian, E. C.; Frey, M.; Fontecilla-Camps, J. C. Crystal Structure of the Nickel-Iron Hydrogenase from Desulfovibrio Gigas. Nature 1995, 373 (6515), 580-587. doi: $10.1038 / 373580 \mathrm{aO}$

(80) Page, C. C.; Moser, C. C.; Chen, X.; Dutton, P. L. Natural Engineering Principles of Electron Tunnelling in Biological Oxidation-Reduction. Nature 1999, 402 (6757), 47-52. doi: 10.1038/46972.

(81) Dementin, S.; Belle, V.; Bertrand, P.; Guigliarelli, B.; Adryanczyk-Perrier, G.; De Lacey, A. L.; Fernandez, V. M.; Rousset, M.; Léger, C. Changing the Ligation of the Distal [4Fe4S] Cluster in NiFe Hydrogenase Impairs Inter- and Intramolecular Electron Transfers. J. Am. Chem. Soc. 2006, 128 (15), 5209-5218. doi: 10.1021/ja060233b.

(82) Zeng, T.; Leimkühler, S.; Wollenberger, U.; Fourmond, V. Transient Catalytic Voltammetry of Sulfite Oxidase Reveals Rate Limiting Conformational Changes. J. Am. Chem. Soc. 2017, 139 (33), 11559-11567. doi: 10.1021/jacs.7b05480.

(83) Cardenas, A. J. P.; Ginovska, B.; Kumar, N.; Hou, J.; Raugei, S.; Helm, M. L.; Appel, A. M.; Bullock, R. M.; O'Hagan, M. Controlling Proton Delivery through Catalyst Structural Dynamics. Angew. Chem. Int. Ed Engl. 2016, 55 (43), 13509-13513. doi: 10.1002/anie.201607460.

(84) Klug, C. M.; Cardenas, A. J. P.; Bullock, R. M.; O'Hagan, M.; Wiedner, E. S. Reversing the Tradeoff between Rate and Overpotential in Molecular Electrocatalysts for H 2 Production. ACS Catal. 2018, 8 (4), 3286-3296. doi: 10.1021/acscatal.7b04379.

(85) Azcarate, I.; Costentin, C.; Robert, M.; Savéant, J.-M. Through-Space Charge Interaction Substituent Effects in Molecular Catalysis Leading to the Design of the Most Efficient Catalyst of $\mathrm{CO}_{2}$-to-CO Electrochemical Conversion. J. Am. Chem. Soc. 2016, 138 (51), 16639-16644. doi: 10.1021/jacs.6b07014.

(86) Dutta, A.; Lense, S.; Hou, J.; Engelhard, M. H.; Roberts, J. A. S.; Shaw, W. J. Minimal Proton Channel Enables $\mathrm{H}_{2}$ Oxidation and Production with a Water-Soluble Nickel-Based Catalyst. J. Am. Chem. Soc. 2013, 135 (49), 18490-18496. doi: 10.1021/ja407826d.

(87) Boralugodage, N. P.; Arachchige, R. J.; Dutta, A.; Buchko, G. W.; Shaw, W. J. Evaluating the Role of Acidic, Basic, and Polar Amino Acids and Dipeptides on a Molecular Electrocatalyst for H 2 Oxidation. Catal. Sci. Technol. 2017, 7 (5), 1108-1121. doi: 10.1039/C6CY02579J. 